\title{
Infrared Spectra of Crystalline Polyphenyls
}

\author{
James E. Stewart ${ }^{1}$ and Max Hellmann
}

\begin{abstract}
Infrared absorption spectra of sixteen selected polyphenyls ranging from biphenyl to $m$-octaphenyl are presented for the 5- to 38-micron region. The observed vibration bands are discussed in terms of a pseudo-symmetry approximation in which the individual rings are assumed to vibrate separately. Indications of interactions between rings are seen in the $\mathrm{C}-\mathrm{H}$ out-of-plane bending vibrations of ortho- and metasubstituted rings. Evidence is given for the existence of two rotational isomers of $m$-quatraphenyl with a free energy difference of about 250 calories per mole.
\end{abstract}

\section{Introduction}

Studies of the vibrational spectra of large molecules have been mostly restricted to empirical correlations of bands with the presence of specific functional groups. Recent publications, however, give evidence of progress in the spectral analysis of large molecules such as polyethylene [1], ${ }^{2}$ substituted polyethylenes [2], and infinite chains of $\left.\mathrm{HgNH}_{2}\right)^{+}$ ions [3]. These substances have the property of possessing translational symmetry and symmetric repeating units, thus reducing the secular equations to a good approximation, from order infinity to some manageable finite order. The homologous series of polyphenyls, $\mathrm{C}_{6} \mathrm{H}_{5}-\left(\mathrm{C}_{6} \mathrm{H}_{4}\right)_{n}-\mathrm{C}_{6} \mathrm{H}_{5}$, have symmetric repeating units, but except for the parapolyphenyls, they have no translational symmetry. However, empirical spectra-structure correlations and analogies with benzene and the substituted benzenes [4] have provided information on the nature of the bands found in the spectra of aromatic compounds and, furthermore, have shown that many of these vibrations are virtually independent of the nature of the substituent but do depend only on its position.

In view of these considerations it seems reasonable to attempt to treat the vibrations of polyphenyls in terms of the symmetry of the individual substituted phenyl units. This is equivalent to replacing the substituents on a given aromatic ring with identical point masses. A pseudosymmetry can then be assigned to the resulting structure and the vibrations of the ring classified under the appropriate symmetry species in the usual way. This paper is a report on an investigation into the feasibility of assigning in this way the infrared absorption bands of a series of polyphenyls containing from 2 to 8 mono- and disubstituted aromatic rings. These compounds have the following advantages: The ring-to-substituent force constants are probably nearly the same in all the compounds; the masses of the substituents are quite large (at least 77 atomic mass units) so the number of phenyl units between a given ring and the last ring in the molecule is not likely to greatly affect its vibrations; and, confusion from overlapping bands of nonphenyl substituents is avoided.

1 Present address: Beckman Instruments, Inc., Fullerton, Calif.

2 Figures in brackets indicate the literature references at the end of this paper,
Randle and Whiffen [5] (referred to hereinafter as $\mathrm{RW}$ ) have analyzed infrared and Raman data from the literature for a large number of substituted single ring aromatic compounds using the pseudosymmetry approximation and have arrived at some very satisfactory assignments. They have also calculated some out-of-plane vibration frequencies for aromatic molecules using the force constants of benzene. More recently, Shimanouchi, Kakiuti, and Gamo [6] have also reported calculations made in this way on single ring compounds.

The polyphenyls were studied in the solid phase, rather than in solution, because it was desired to observe, if possible, the contributions of the individual units of a polyphenyl chain to the characteristic absorption bands. In liquids, the variations in environment tend to broaden absorption bands until the finer structure is lost. On the other hand, the analysis of crystal phase spectra is often complicated by the occurrence of lattice vibrations and the strengthening of combination vibrations and splitting of bands by intermolecular vibrational interactions.

\section{Experimental Details}

Infrared spectra of 16 polyphenyls are shown in figures 1 through 8 . The frequencies of some of the bands and their assignments are given in table 1 . A Perkin-Elmer model 21 spectrophotometer was used for the measurements. The instrument was equipped with a sodium chloride prism for use in the 5- to $15-\mu$ region and a cesium bromide prism for the 15- to $38-\mu$ region. Reststrahlen filter plates of lithium fluoride and calcium fluoride were used to control stray radiation in the $15-$ to $30-\mu$ and $30-$ to $38-\mu$ regions, respectively. Stray radiation did not exceed 2 percent.

Samples were prepared in the form of alkali halide pellets using the die and procedure reported earlier [7]. Potassium iodide was found to be most satisfactory in reducing scattered energy losses, but even so, scatter losses at shorter wavelengths were sometimes excessive. No attempt was made to compensate for scatter or reflection losses. A satisfactory spectrum of biphenyl in pellet form was not obtained in the 5 - to $15-\mu$ region. The spectrum shown is of a specimen of biphenyl melted and recrystallized between sodium chloride windows separated with a 


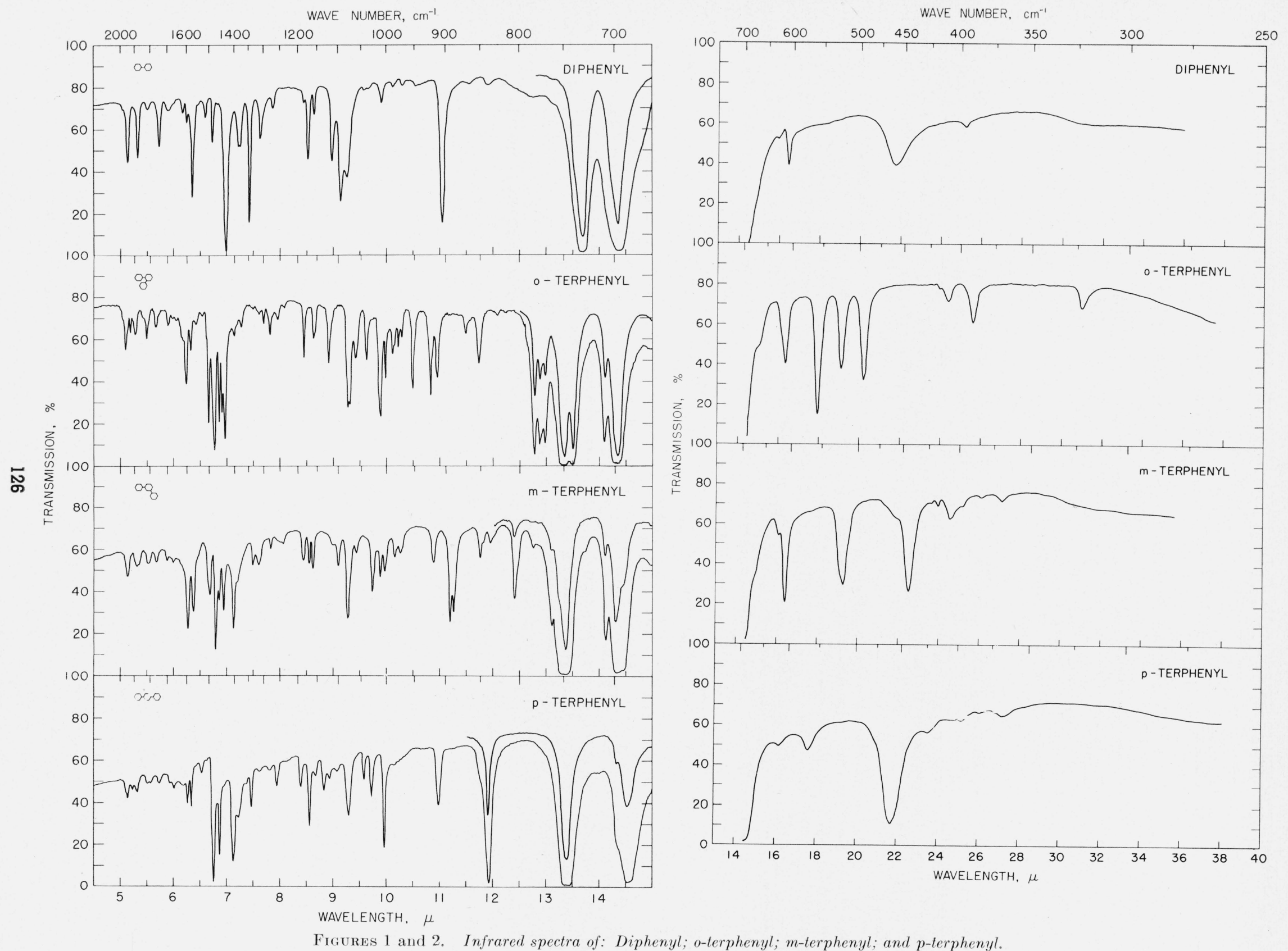



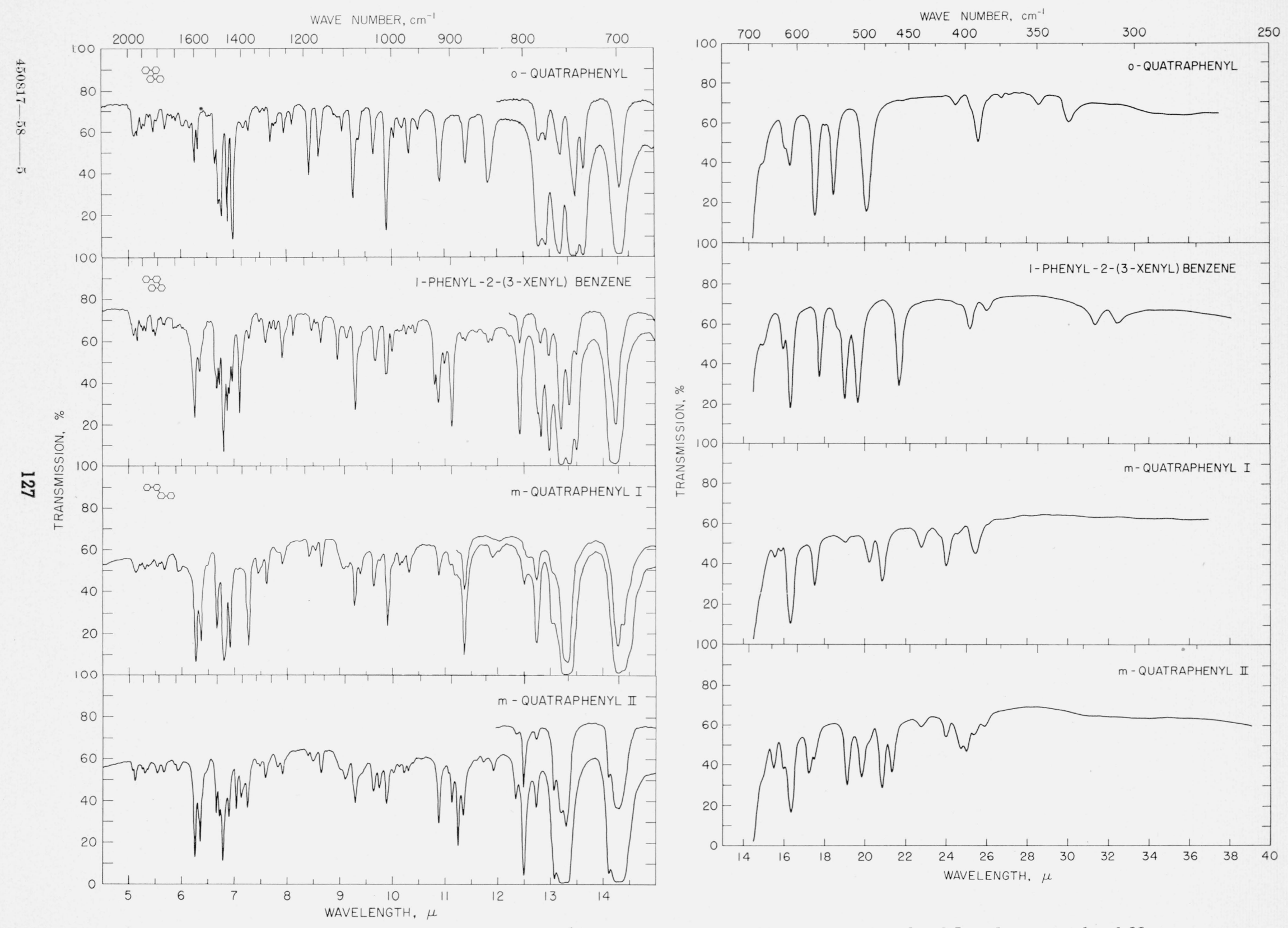

FIGURES 3 and 4. Infrared spectra of: o-Quatraphenyl; 1-phenyl-2-(3-xenyl)benzene; m-quatraphenyl I; and m-quatraphenyl II. 

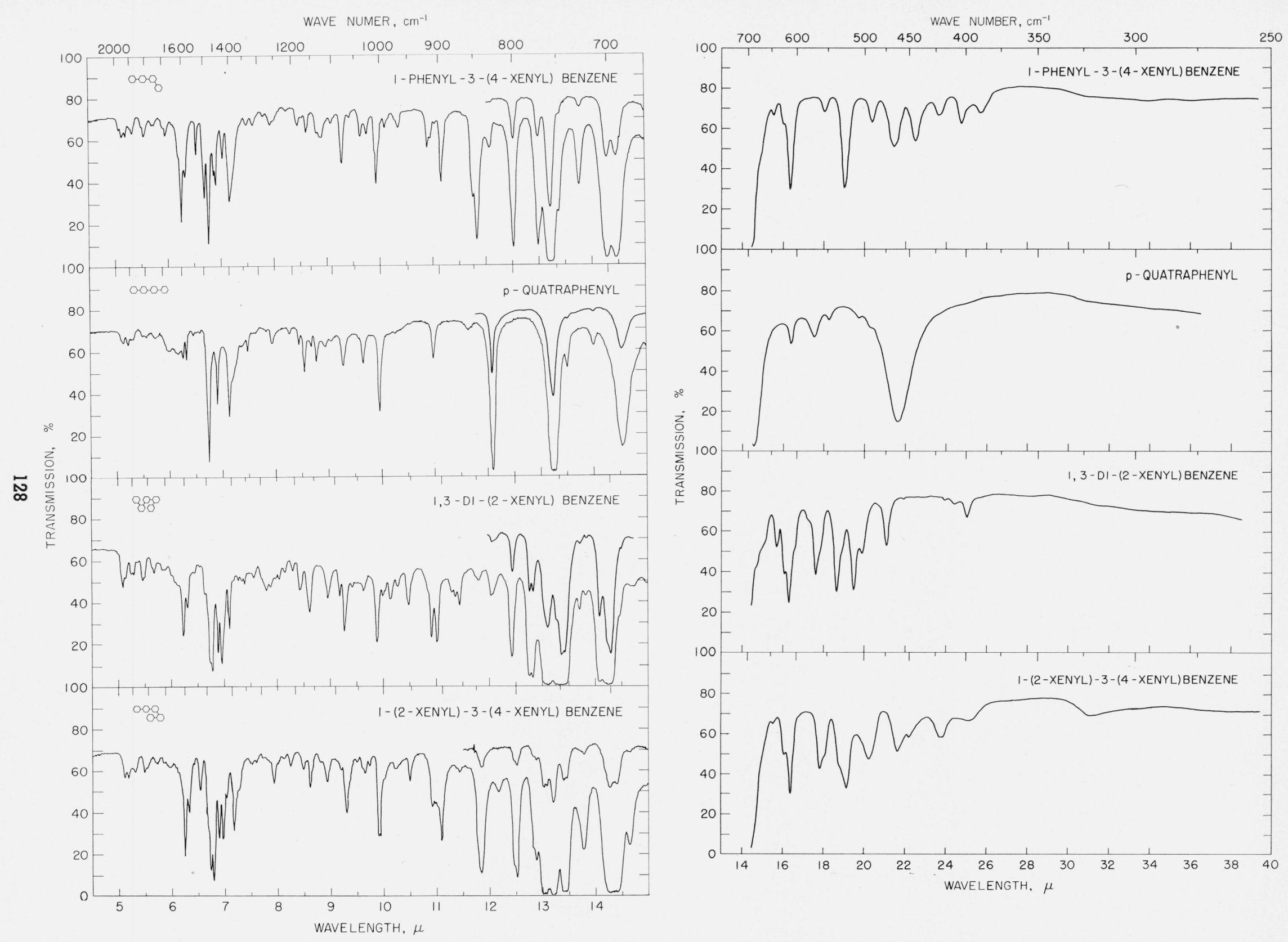

Figures 5 and $6 . \quad$ Infrared spectra of: 1-Phenyl-3-(4-xenyl) benzene; p-quatraphenyl; 1,3-di-(2-xenyl) benzene; and 1-(2-xenyl)-3-(4-xenyl) benzene. 


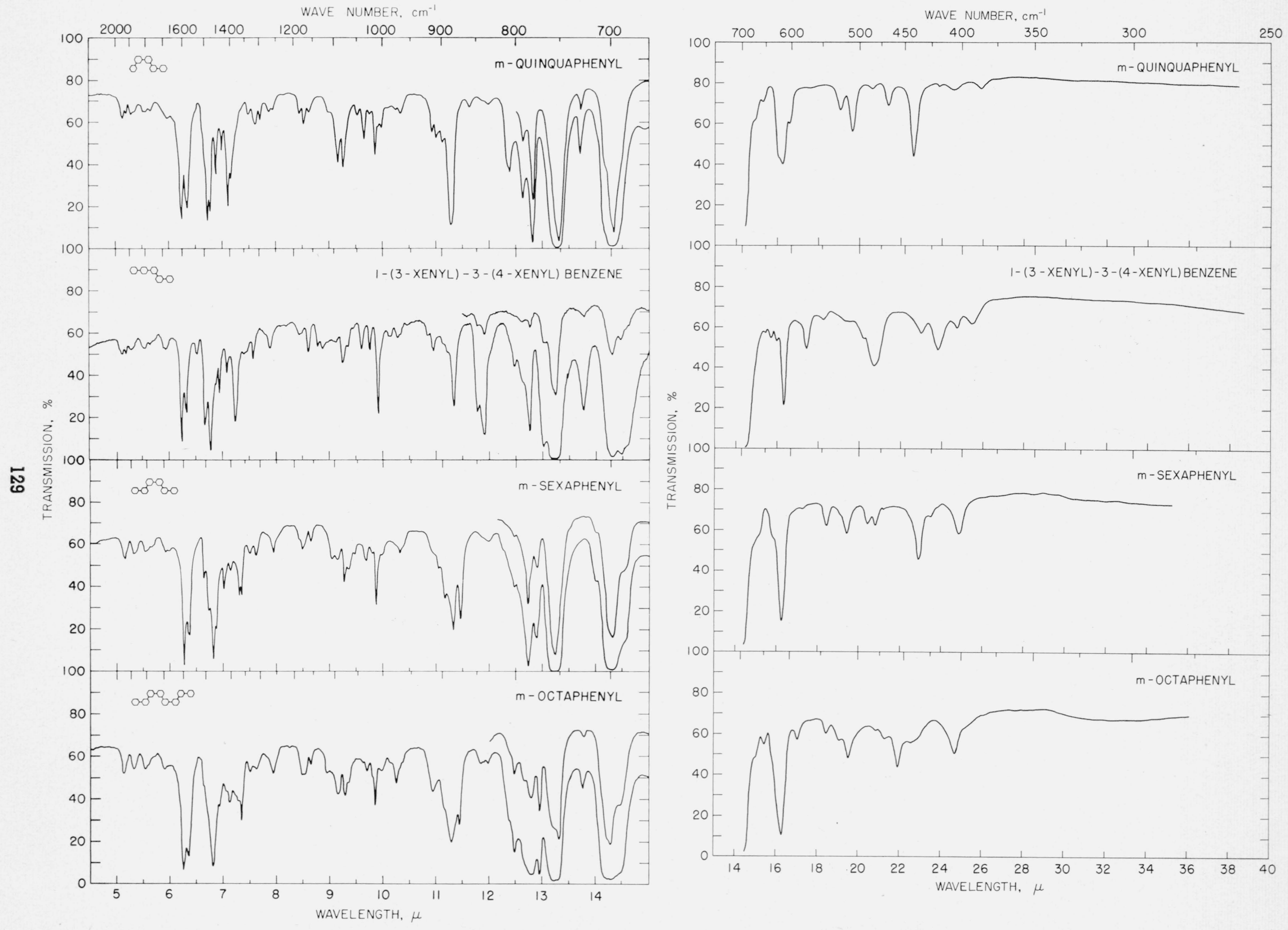

FIGURES 7 and 8. Infrared spectra of: m-Quinquaphenyl; 1-(3-xenyl)-3-(4-xenyl) benzene; m-sexaphenyl; and m-octaphenyl. 
TABLE 1. Frequencies and assignments of some bands in the infrared spectra of polyphenyls

M, o, m, p refer to mono-, ortho-, meta-, and parasubstituted rings. $\|\mathrm{H},\| \mathrm{C}$, $\perp \mathrm{H}$, and $\perp \mathrm{C}$ refer to vibrations which are primarily in-plane $\mathrm{CH}$, in-plane CCC, out-of-plane $\mathrm{CH}$, and out-of-plane $\mathrm{CCC}$ bending motions. The letters in paren$v$, very; s, strong; $m$, medium; w, weak; sh, shoulder.

\begin{tabular}{|c|c|c|c|c|c|}
\hline \multicolumn{6}{|c|}{ Biphenyl } \\
\hline $\begin{array}{r}c m^{-1} \\
397 \\
460 \\
609 \\
626 \\
\\
696 \\
730 \\
835 \\
903\end{array}$ & $\begin{array}{c}\mathrm{vw} \\
\mathrm{m} \\
\mathrm{m} \\
\mathrm{vw} \\
\\
\mathrm{vs} \\
\mathrm{vs} \\
\mathrm{vw} \\
\mathrm{m}\end{array}$ & $\begin{array}{l}\mathrm{M}\left(A_{2}\right) \perp \mathrm{C} \\
\mathrm{M}\left(B_{2}\right) \perp \mathrm{C} \\
\mathrm{M}\left(B_{1}\right) \| \mathrm{C} \\
\mathrm{M}\left(A_{1}\right) \| \mathrm{C} \\
\mathrm{M}\left(B_{2}\right) \perp \mathrm{C} \\
\mathrm{M}\left(B_{2}\right) \perp \mathrm{H} \\
\mathrm{M}\left(A_{2}\right) \perp \mathrm{H} \\
\mathrm{M}\left(B_{2}\right) \perp \mathrm{H}\end{array}$ & $\begin{array}{r}c m^{-1} \\
970 \\
987 \\
1,006 \\
1,042 \\
1,078 \\
1,092 \\
1,112 \\
1,168 \\
1,182\end{array}$ & $\begin{array}{l}\text { vvw } \\
\text { vvw } \\
\mathrm{m} \\
\mathrm{m} \\
\mathrm{m} \\
\mathrm{m} \\
\mathrm{w} \\
\mathrm{m} \\
\mathrm{w}\end{array}$ & $\begin{array}{l}\text { M }\left(A_{2}\right), \mathrm{M}\left(B_{2}\right) \perp \mathrm{H} \\
\mathrm{M}\left(A_{1}\right) \| \mathrm{C} \\
\mathrm{M}\left(A_{1}\right) \| \mathrm{H} \\
\mathrm{M}\left(B_{1}\right) \| \mathrm{H} \\
\mathrm{M}\left(B_{1}\right) \mathrm{H} \| ? \\
\mathrm{M}\left(B_{1}\right) \| \mathrm{H} \\
\mathrm{M}\left(A_{1}\right) \| \mathrm{H}\end{array}$ \\
\hline \multicolumn{6}{|c|}{$o$-Terphenyl } \\
\hline $\begin{array}{l}391 \\
410 \\
495 \\
524 \\
557 \\
\\
612 \\
619 \\
697 \\
709 \\
741\end{array}$ & $\begin{array}{c}\mathrm{m} \\
\mathrm{w} \\
\mathrm{m} \\
\mathrm{s} \\
\mathrm{m} \\
\mathrm{m} \\
\mathrm{sh} ? \\
\mathrm{vs} \\
\mathrm{s} \\
\mathrm{vs}\end{array}$ & $\begin{array}{l}\mathrm{O}\left(A_{2}\right), \mathrm{M}\left(A_{2}\right) \perp \mathrm{C} \\
\mathrm{O} \\
\mathrm{M}\left(B_{1}\right), 0\left(B_{1}\right) \| \mathrm{C} \\
\mathrm{M}\left(A_{1}\right) \| \mathrm{C} \\
\mathrm{M}\left(B_{2}\right) \perp \mathrm{C} \\
\mathrm{o}\left(A_{2}\right) \perp \mathrm{C} \\
\mathrm{M}\left(B_{2}\right) \perp \mathrm{H} \\
\mathrm{O}\left(B_{2}\right) \perp \mathrm{H}\end{array}$ & $\begin{array}{r}852 \\
870 \\
912 \\
922 \\
953 \\
\\
1,000 \\
1,010 \\
1,038 \\
1,060 \\
1,073 \\
1,077 \\
1,120 \\
1,157 \\
1,182\end{array}$ & $\begin{array}{c}\mathrm{m} \\
\mathrm{w} \\
\mathrm{s} \\
\mathrm{s} \\
\mathrm{s} \\
\mathrm{m} \\
\mathrm{s} \\
\mathrm{m} \\
\mathrm{m} \\
\mathrm{s} \\
\mathrm{s} \\
\mathrm{m} \\
\mathrm{m} \\
\mathrm{m}\end{array}$ & $\begin{array}{l}\mathrm{o}\left(A_{2}\right) \perp \mathrm{H} \\
\mathrm{o}\left(A_{2}\right) \perp \mathrm{H} \\
\mathrm{o}\left(B_{2}\right) \perp \mathrm{H} \\
\mathrm{M}\left(B_{2}\right) \perp \mathrm{H} \\
\mathrm{M}\left(A_{1}\right), \mathrm{o}\left(B_{1}\right) \| \mathrm{C} \\
\mathrm{M}\left(A_{1}\right) \| \mathrm{H} \\
\mathrm{o}\left(A_{1}\right) \| \mathrm{H} \\
\mathrm{M}\left(B_{1}\right) \| \mathrm{H} \\
\mathrm{o}\left(B_{1}\right) \| \mathrm{H} \\
\mathrm{M}\left(B_{1}\right), \mathrm{o}\left(A_{1}\right) \| \mathrm{H} \\
\mathrm{o}\left(B_{1}\right) \| \mathrm{H}\end{array}$ \\
\hline \multicolumn{6}{|c|}{$m$-Terphenyl } \\
\hline $\begin{array}{l}408 \\
444 \\
455 \\
519 \\
610 \\
\\
622 \\
692 \\
698 \\
708 \\
748 \\
806\end{array}$ & $\begin{array}{l}\mathrm{w} \\
\mathrm{sh} \\
\mathrm{vs} \\
\mathrm{m} \\
\mathrm{vs} \\
\mathrm{m}\end{array}$ & $\begin{array}{l}\mathrm{m}\left(A_{2}\right), \mathrm{M}\left(A_{2}\right) \perp \mathrm{C} \\
\mathrm{m} \\
\mathrm{M}\left(B_{2}\right) \perp \mathrm{C} \\
\mathrm{m}\left(B_{1}\right), \mathrm{m}\left(B_{1}\right) \| \mathrm{C} \\
\mathrm{M}\left(A_{1}\right) \| \mathrm{C} \\
\mathrm{M}\left(B_{2}\right), \mathrm{m}\left(B_{2}\right) \perp \mathrm{C} \\
\mathrm{M}\left(B_{2}\right) \perp \mathrm{H} \\
\mathrm{m}\left(B_{2}\right) \perp \mathrm{H}\end{array}$ & $\begin{array}{r}880 \\
893 \\
918 \\
974 \\
1,003 \\
1,011 \\
1,027 \\
1,090 \\
1,160 \\
1,170 \\
1,185\end{array}$ & $\begin{array}{l}\mathrm{m} \\
\mathrm{m} \\
\mathrm{w} \\
\mathrm{w} \\
\mathrm{w} \\
\mathrm{w} \\
\mathrm{m} \\
\mathrm{m} \\
\mathrm{w} \\
\mathrm{w} \\
\mathrm{w}\end{array}$ & $\begin{array}{l}\mathrm{m}\left(B_{2}\right) \perp \mathrm{H} \\
\mathrm{M}\left(B_{2}\right), \mathrm{m}\left(A_{2}\right) \perp \mathrm{H} \\
\mathrm{m}\left(B_{2}\right) \perp \mathrm{H} \\
\mathrm{M}\left(A_{1}\right), \mathrm{m}\left(A_{1}\right) \| \mathrm{C} \\
\mathrm{m}\left(B_{1}\right), \mathrm{M}\left(A_{1}\right) \| \mathrm{H} \\
\mathrm{m}\left(A_{1}\right), \mathrm{M}\left(B_{1}\right) \| \mathrm{H} \\
\mathrm{M}\left(B_{1}\right) \| \mathrm{H} \\
\mathrm{m}\left(B_{1}\right) \| \mathrm{H} \\
\mathrm{M}\left(A_{1}\right), \mathrm{m}\left(B_{1}\right) \| \mathrm{H}\end{array}$ \\
\hline \multicolumn{6}{|c|}{$p$-Terphenyl } \\
\hline $\begin{array}{l}397 \\
460 \\
618 \\
688 \\
698 \\
\\
746 \\
838 \\
850 \\
911\end{array}$ & $\begin{array}{l}\text { w } \\
\text { S } \\
\text { W } \\
\text { vs } \\
\text { vs } \\
\text { vs } \\
\text { vs } \\
\text { sh } \\
\text { m }\end{array}$ & $\begin{array}{l}\mathrm{p}\left(A_{\mu}\right), \mathrm{M}\left(A_{2}\right) \perp \mathrm{C} \\
\mathrm{p}\left(B_{1} \mu\right), \mathrm{M}\left(B_{2}\right) \perp \mathrm{C} \\
\mathrm{M}\left(B_{1}\right) \| \mathrm{C} \\
\mathrm{p}\left(B_{3 \mathrm{~g}}\right), \mathrm{M}\left(B_{2}\right) \perp \mathrm{C} \\
\mathrm{M}\left(B_{2}\right) \perp \mathrm{H} \\
\mathrm{p}\left(B_{1} \mu\right) \perp \mathrm{H} \\
\mathrm{p}\left(B_{2 \mathrm{~g}}\right) \perp \mathrm{H} \\
\mathrm{M}\left(B_{2}\right), \mathrm{p}(A \mu \text { or } \\
\left.B_{3 \mathrm{~g}}\right) \perp \mathrm{H}\end{array}$ & $\begin{array}{l}1,003 \\
1,028 \\
1,043 \\
1,075 \\
1,083 \\
1,132 \\
1,168 \\
1,190\end{array}$ & $\begin{array}{l}\mathrm{s} \\
\mathrm{W} \\
\mathrm{W} \\
\mathrm{m} \\
\mathrm{w} \\
\mathrm{W} \\
\mathrm{m} \\
\mathrm{w}\end{array}$ & $\begin{array}{l}\mathrm{p}\left(B_{2} \mu\right), \mathrm{M}\left(A_{1}\right) \| \mathrm{C} \\
\mathrm{p}\left(B_{2}\right) \| \mathrm{H} \\
\mathrm{M}\left(A_{1}\right) \| \mathrm{H} \\
\mathrm{M}\left(B_{1}\right) \| \mathrm{H} \\
\mathrm{p}\left(B_{3 \mu}\right) \| \mathrm{H} \\
\mathrm{p}\left(A_{\mathrm{g}}\right), \mathrm{M}\left(B_{1}\right) \| \mathrm{H} \\
\mathrm{p}\left(B_{1 \mathrm{~g}}\right) \| \mathrm{H}\end{array}$ \\
\hline \multicolumn{6}{|c|}{ o-Quatraphenyl } \\
\hline $\begin{array}{l}390 \\
409 \\
498 \\
541 \\
569 \\
613 \\
\\
622 \\
698 \\
733 \\
741 \\
757\end{array}$ & $\begin{array}{c}\mathrm{m} \\
\mathrm{w} \\
\mathrm{s} \\
\mathrm{s} \\
\mathrm{s} \\
\mathrm{m}\end{array}$ & $\left\{\begin{array}{l}\mathrm{o}\left(A_{2}\right), \mathrm{M}\left(A_{2}\right) \perp \mathrm{C} \\
\mathrm{O} \\
\mathrm{M}\left(B_{1}\right), 0\left(B_{1}\right) \| \mathrm{C} \\
\mathrm{M}\left(A_{1}\right) \| \mathrm{C} \\
\mathrm{M}\left(B_{2}\right), 0\left(A_{2}\right) \perp \mathrm{C} \\
\mathrm{M}\left(B_{2}\right) \perp \mathrm{H}\end{array}\right.$ & $\begin{array}{r}773 \\
781 \\
876 \\
916 \\
968 \\
1,010 \\
1,035 \\
1,066 \\
1,078 \\
1,103 \\
1,159 \\
1,185\end{array}$ & $\begin{array}{l}\mathrm{vs} \\
\mathrm{vs} \\
\mathrm{m} \\
\mathrm{m} \\
\mathrm{w} \\
\mathrm{s} \\
\mathrm{m} \\
\mathrm{w} \\
\mathrm{m} \\
\mathrm{w} \\
\mathrm{m} \\
\mathrm{m}\end{array}$ & $\begin{array}{l}\} \mathrm{o}\left(B_{2}\right) \perp \mathrm{H} \\
\mathrm{o}\left(A_{2}\right) \perp \mathrm{H} \\
\mathrm{o}\left(B_{2}\right), \mathrm{M}\left(B_{2}\right) \perp \mathrm{H} \\
\mathrm{o}\left(A_{2}\right) \perp \mathrm{H} \\
\mathrm{o}\left(B_{1}\right) \| \mathrm{C} \\
\mathrm{M}\left(A_{1}\right) \| \mathrm{H} \\
\mathrm{o}\left(A_{1}\right) \| \mathrm{H} \\
\mathrm{M}\left(B_{1}\right) \| \mathrm{H} \\
\mathrm{o}\left(B_{1}\right) \| \mathrm{H} \\
\mathrm{o}\left(A_{1}\right) \| \mathrm{H} \\
\mathrm{o}\left(B_{1}\right) \| \mathrm{H}\end{array}$ \\
\hline
\end{tabular}

TABLE 1. Frequencies and assignments of some bands in the infrared spectra of polyphenyls - Continued

\begin{tabular}{|c|c|c|c|c|c|}
\hline \multicolumn{6}{|c|}{ 1-Phenyl-2-(3-xenyl) benzene } \\
\hline $\begin{array}{l}525 \\
563 \\
613 \\
626 \\
669 \\
\\
702 \\
740 \\
748 \\
757 \\
770 \\
783\end{array}$ & $\begin{array}{l}\text { VS } \\
\text { W } \\
\text { VS } \\
\text { VS } \\
\text { S } \\
\text { W }\end{array}$ & $\begin{array}{l}\text { M }\left(A_{2}\right), \mathrm{o}\left(A_{2}\right), \\
\mathrm{m}\left(A_{2}\right) \perp \mathrm{C} \\
\mathrm{m}, \mathrm{M}\left(B_{2}\right) \\
\mathrm{o}\end{array}$ & $\begin{array}{r}c m^{-1} \\
804 \\
878 \\
897 \\
908 \\
918 \\
925 \\
959 \\
975 \\
1,001 \\
1,011 \\
1,013 \\
1,034 \\
1,076 \\
1,094 \\
1,116 \\
1,170 \\
1,181\end{array}$ & $\begin{array}{c}\mathrm{S} \\
\mathrm{W} \\
\mathrm{S} \\
\mathrm{w} \\
\mathrm{m} \\
\mathrm{m} \\
\mathrm{W} \\
\mathrm{W} \\
\mathrm{W} \\
\mathrm{m} \\
\mathrm{m} \\
\mathrm{m} \\
\mathrm{S} \\
\mathrm{S} \\
\mathrm{W} \\
\mathrm{m} \\
\mathrm{W} \\
\\
\mathrm{W}\end{array}$ & $\begin{array}{l}\mathrm{m}\left(B_{2}\right) \perp \mathrm{H} \\
\mathrm{o}\left(A_{2}\right) \perp \mathrm{H} \\
\mathrm{m}\left(B_{2}\right) \perp \mathrm{H} \\
\mathrm{M}\left(B_{2}\right) \perp \mathrm{H} \\
\mathrm{m}\left(A_{2}\right) \perp \mathrm{H} \\
\mathrm{o}\left(B_{2}\right) \perp \mathrm{H} \\
\mathrm{m}\left(B_{2}\right) \perp \mathrm{H} \\
\mathrm{o}\left(A_{2}\right) \perp \mathrm{H} \\
\mathrm{M}\left(A_{1}\right), \mathrm{o}\left(B_{1}\right), \\
\mathrm{m}\left(A_{1}\right) \| \mathrm{C} \\
\mathrm{o}\left(A_{1}\right), \mathrm{m}\left(B_{1}\right), \\
\mathrm{M}\left(A_{1}\right) \| \mathrm{H} \\
\mathrm{M}\left(B_{1}\right) \| \mathrm{H} \\
\mathrm{m}\left(A_{1}\right) \| \mathrm{H} \\
\mathrm{o}\left(B_{1}\right) \| \mathrm{H} \\
\mathrm{M}\left(B_{1}\right), \mathrm{o}\left(A_{1}\right), \\
\mathrm{m}\left(B_{1}\right) \| \mathrm{H} \\
\mathrm{o}\left(B_{1}\right), \mathrm{m}\left(B_{1}\right) \| \mathrm{H}\end{array}$ \\
\hline \multicolumn{6}{|c|}{ m-Quatraphenyl I } \\
\hline $\begin{array}{l}525 \\
571 \\
612 \\
631 \\
\\
642 \\
690 \\
695 \\
700\end{array}$ & $\begin{array}{l}\text { W } \\
\text { sh } \\
\text { vs } \\
\text { vs }\end{array}$ & $\begin{array}{l}\int_{\mathrm{m}}\left(A_{2}\right), \mathrm{M}\left(A_{2}\right) \perp \mathrm{C} \\
\mathrm{m}\left(B_{2}\right) \\
\mathrm{m}\left(B_{1}\right), \mathrm{M}\left(B_{1}\right) \| \mathrm{C} \\
\mathrm{M}\left(A_{1}\right) \| \mathrm{C} \\
\mathrm{m}\left(A_{1}\right) \| \mathrm{C} \\
\mathrm{M}\left(B_{2}\right), \mathrm{m}\left(B_{2}\right) \perp \mathrm{C}\end{array}$ & $\begin{array}{r}751 \\
767 \\
784 \\
801 \\
880 \\
919 \\
969 \\
1,010 \\
1,037 \\
1,066 \\
1,078 \\
1,156 \\
1,172 \\
1,189\end{array}$ & $\begin{array}{l}\text { vs } \\
\text { vs } \\
\text { S } \\
\text { m } \\
\text { s } \\
\text { W } \\
\text { W } \\
\text { m } \\
\text { m } \\
\text { W } \\
\text { m } \\
\text { W } \\
\text { W } \\
\text { W }\end{array}$ & $\begin{array}{l}\left\{\mathrm{M}\left(B_{2}\right) \perp \mathrm{H}\right. \\
\mathrm{m}\left(B_{2}\right) \perp \mathrm{H} \\
\mathrm{m}\left(B_{2}\right) \perp \mathrm{H} \\
\mathrm{m}\left(A_{2}\right), \mathrm{M}\left(B_{2}\right) ? \perp \mathrm{H} \\
\mathrm{m}\left(B_{2}\right) \perp \mathrm{H} \\
\mathrm{m}\left(A_{1}\right), \mathrm{M}\left(A_{1}\right) \| \mathrm{C} \\
\mathrm{m}\left(B_{1}\right), \mathrm{M}\left(A_{1}\right) \| \mathrm{H} \\
\mathrm{m}\left(A_{1}\right), \mathrm{M}\left(B_{1}\right) \| \mathrm{H} \\
\mathrm{M}\left(B_{1}\right) \| \mathrm{H} \\
\mathrm{m}\left(B_{1}\right) \mathrm{H} \\
\mathrm{m}\left(B_{1}\right) \| \mathrm{H}\end{array}$ \\
\hline
\end{tabular}

m-Quatraphenyl II

\begin{tabular}{|c|c|c|c|c|c|}
\hline 386 & w & & 799 & $\mathrm{~s}$ & \\
\hline 394 & w & & 810 & $\mathrm{~m}$ & $\mathrm{~m}\left(B_{2}\right) \perp \mathrm{H}$ \\
\hline 400 & $\mathrm{~m}$ & $\mathrm{M}\left(A_{2}, \mathrm{~m}\left(A_{2}\right) \perp \mathrm{C}\right.$ & 888 & $\mathrm{~s}$ & $\mathrm{~m}\left(B_{2}\right) \perp \mathrm{H}$ \\
\hline $\begin{array}{l}404 \\
417\end{array}$ & $\begin{array}{l}\text { W } \\
\text { W }\end{array}$ & & $\begin{array}{l}898 \\
920\end{array}$ & $\underset{\mathrm{s}}{\mathrm{m}}$ & $\mathrm{m}\left(A_{2}\right), \mathrm{M}\left(B_{2}\right) \perp \mathrm{H}$ \\
\hline $\begin{array}{l}439 \\
469\end{array}$ & $\mathrm{w}$ & $\mathrm{m}, \mathrm{M}\left(B_{2}\right) \perp \mathrm{C}$ & 971 & W & $\mathrm{m}\left(B_{2}\right) \perp \mathrm{H}$ \\
\hline 504 & $\mathrm{~m}$ & 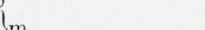 & 1,012 & $\mathrm{~m}$ & $\mathrm{~m}\left(A_{1}\right), \mathrm{M}\left(A_{1}\right) \| \mathrm{C}$ \\
\hline 523 & $\mathrm{~m}$ & $\mathrm{~m}$ & 1,026 & $\mathrm{w}$ & $\mathrm{m}\left(B_{1}\right) \| \mathrm{H}$ \\
\hline 571 & $\mathrm{w}$ & & 1,038 & w & $\mathrm{M}\left(A_{1}\right) \| \mathrm{H}$ \\
\hline 579 & $\mathrm{~m}$ & & 1,076 & $\mathrm{~m}$ & $\mathrm{~m}\left(A_{1}\right) \| \mathrm{H}$ \\
\hline 611 & $\mathrm{~m}$ & $\mathrm{~m}\left(B_{1}\right), \mathrm{M}\left(B_{1}\right) \| \mathrm{C}$ & 1,099 & $\mathrm{w}$ & $\mathrm{M}\left(B_{1}\right) \| \mathrm{H}$ \\
\hline 625 & W & $\mathrm{M}\left(A_{1}\right) \| \mathrm{C}$ & 1,105 & $\mathrm{w}$ & $\int \mathrm{N}\left(\mathrm{B}_{1}\right)$ \\
\hline $\begin{array}{l}645 \\
699\end{array}$ & $\begin{array}{l}\text { W } \\
\text { vs }\end{array}$ & $\mathrm{m}\left(A_{1}\right) \| \mathrm{C}$ & $\begin{array}{l}1,157 \\
1,179\end{array}$ & $\begin{array}{l}\mathrm{W} \\
\mathrm{W}\end{array}$ & $\begin{array}{l}\mathrm{M}\left(B_{1}\right) \| \mathrm{H} \\
\mathrm{m}\left(B_{1}\right) \| \mathrm{H}\end{array}$ \\
\hline 701 & vs & $\mathrm{M}\left(B_{2}\right), \mathrm{m}\left(B_{2}\right) \perp \mathrm{C}$ & 1,192 & w & $\mathrm{m}\left(B_{1}\right), \mathrm{M}\left(A_{1}\right) \| \mathrm{H}$ \\
\hline 708 & vs & & & & \\
\hline 752 & vs & & & & \\
\hline 756 & vs & $\mathrm{M}\left(B_{2}\right) \perp \mathrm{H}$ & & & \\
\hline 765 & vS & & & & \\
\hline
\end{tabular}

1-Phenyl-3-(4-xenyl) benzene

\begin{tabular}{|c|c|c|c|c|c|}
\hline 399 & $\mathrm{~W}$ & $\mathrm{M}\left(A_{0}\right) \mathrm{m}\left(A_{0}\right)$ & 799 & & $m\left(R_{0}\right)$ \\
\hline 403 & $\mathrm{~m}$ & $\mathrm{p}(A, \mathrm{C}$ & 845 & s & $\mathrm{p}\left(\mathrm{B}_{1}\right) \perp \mathrm{H}$ \\
\hline 443 & $\mathrm{~m}$ & $\mathrm{~m}$ & 851 & $\mathrm{~m}$ & $\mathrm{p}\left(B_{2 \mathrm{~g}}\right) \perp \mathrm{H}$ \\
\hline 465 & $\mathrm{~m}$ & $\mathrm{p}\left(B_{1 \mathrm{u}}\right), \mathrm{M}\left(B_{2}\right) \perp \mathrm{C}$ & 896 & $\mathrm{~m}$ & $\mathrm{~m}\left(B_{2}\right) \perp \mathrm{H}$ \\
\hline 525 & $\mathrm{~s}$ & $\mathrm{~m}$ & 918 & w & $\begin{array}{l}\mathrm{m}\left(A_{2}\right), \mathrm{p}\left(A_{\mathrm{u}} ; B_{3 \mathrm{~g}}\right), \\
\mathrm{M}\left(B_{2}\right) \perp \mathrm{H}\end{array}$ \\
\hline 553 & w & & & & \\
\hline 611 & $\mathrm{~s}$ & $\mathrm{~m}\left(B_{1}\right), \mathrm{M}\left(B_{1}\right) \| \mathrm{C}$ & 966 & W & $\mathrm{m}\left(B_{2}\right) \perp \mathrm{H}$ \\
\hline $\begin{array}{l}623 \\
642\end{array}$ & $\begin{array}{l}\mathrm{W} \\
\mathrm{W}\end{array}$ & $\begin{array}{l}\mathrm{M}\left(A_{1}\right) \| \mathrm{C} \\
\mathrm{m}\left(A_{1}\right) \| \mathrm{C}\end{array}$ & 1,007 & $\mathrm{~m}$ & $\begin{array}{c}\mathrm{M}\left(A_{1}\right), \mathrm{m}\left(A_{1}\right), \\
\mathrm{p}(B, \ldots) \| \mathrm{C}\end{array}$ \\
\hline 691 & vs & $\mathrm{m}\left(A_{1}\right) \| \mathrm{C}$ & 1,026 & w & $\mathrm{m}\left(B_{1}\right), \mathrm{p}\left(B_{2 \mathrm{u}}\right) \| \mathrm{H}$ \\
\hline 700 & vs & $\mathrm{M}\left(B_{2}\right), \mathrm{m}\left(B_{2}\right) \perp \mathrm{C}$ & 1,038 & $\mathrm{~W}$ & $\mathrm{M}\left(A_{1}\right) \| \mathrm{H}$ \\
\hline 748 & $\mathrm{VW}$ & & 1,077 & $\mathrm{~m}$ & $\mathrm{~m}\left(A_{1}\right), \mathrm{M}\left(B_{1}\right) \| \mathrm{H}$ \\
\hline 757 & vs & $\mathrm{M}\left(\mathrm{B}_{2}\right) \perp \mathrm{H}$ & 1,136 & $\mathrm{w}$ & $\mathrm{p}\left(B_{3 \mathrm{u}}\right) \| \mathrm{H}$ \\
\hline 770 & $\mathrm{~s}$ & & 1,161 & $\mathrm{w}$ & $\mathrm{m}\left(B_{1}\right), \mathrm{p}\left(A_{\mathrm{g}}\right)$ \\
\hline & & & 1,183 & w & $\mathrm{m}\left(B_{1}\right), \mathrm{p}\left(B_{1 \mathrm{~g}}\right), \| \mathrm{H}$ \\
\hline
\end{tabular}


TABLE 1. Frequencies and assignments of some bands in the infrared spectra of polyphenyls - Continued

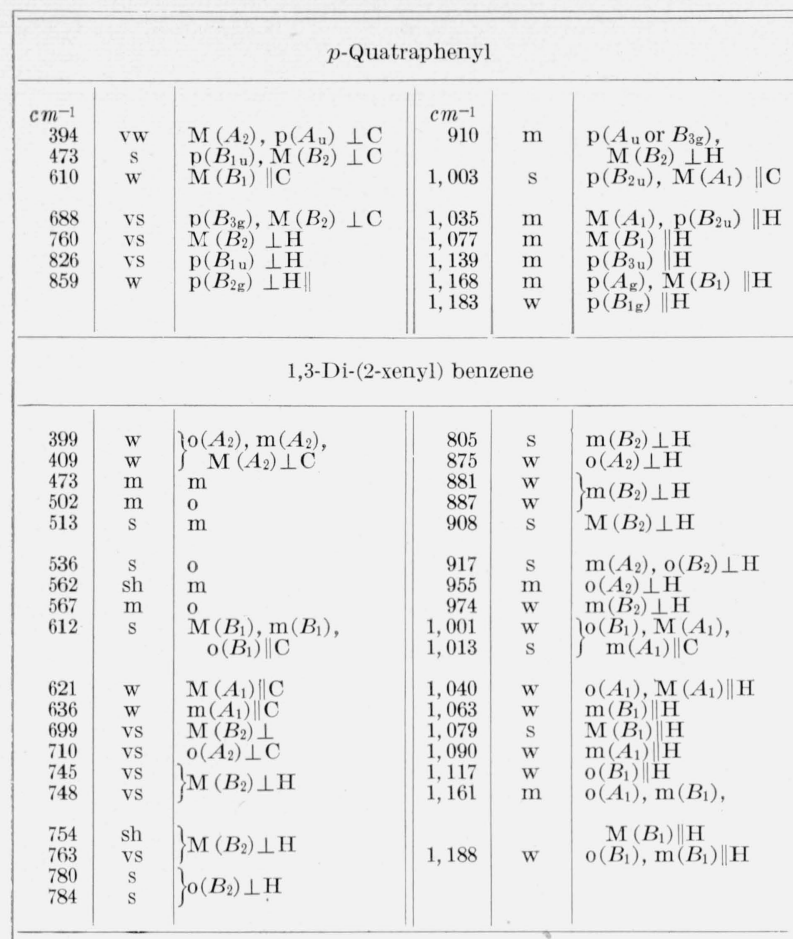

1-(2-Xenyl)-3-(4-xenyl) benzene

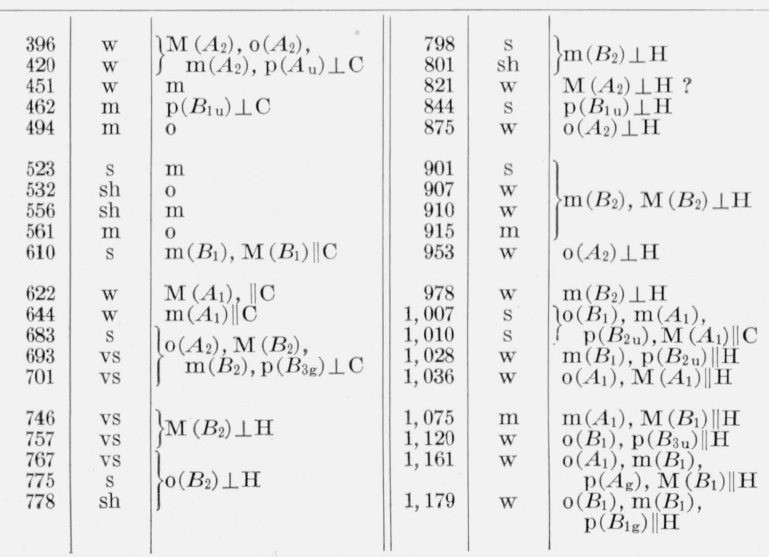

m-Quinquaphenyl

\begin{tabular}{|c|c|c|c|c|c|}
\hline 394 & w & & 886 & $\mathrm{~s}$ & \\
\hline 406 & $\mathrm{w}$ & $\mathrm{m}\left(A_{2}\right), \mathrm{M}\left(A_{2}\right) \perp \mathrm{C}$ & 898 & $\mathrm{w}$ & $\mathrm{M}\left(B_{2}\right) \perp \mathrm{H}$ \\
\hline 418 & $\mathrm{VW}$ & & 907 & w & \\
\hline 441 & $\mathrm{~s}$ & $\mathrm{~m}$ & 914 & $\mathrm{w}$ & $\mathrm{m}\left(A_{2}\right), \mathrm{M}\left(B_{2}\right) \perp \mathrm{H}$ \\
\hline 466 & $\mathrm{w}$ & $\mathrm{M}\left(B_{2}\right) \perp \mathrm{C}$ & 967 & $\mathrm{~W}$ & $\mathrm{~m}\left(B_{2}\right) \perp \mathrm{H}$ \\
\hline 508 & $\mathrm{~m}$ & $\mathrm{~m}$ & $\begin{array}{l}1,002 \\
1,013\end{array}$ & $\begin{array}{l}\mathrm{W} \\
\mathrm{m}\end{array}$ & $\mathrm{m}\left(A_{1}\right), \mathrm{M}\left(A_{1}\right) \| \mathrm{C}$ \\
\hline 568 & VW & & 1,024 & $\mathrm{~m}$ & $m(B) H$ \\
\hline 614 & $\mathrm{~s}$ & $\mathrm{~m}\left(B_{1}\right), \mathrm{M}\left(B_{1}\right) \| \mathrm{C}$ & 1,035 & $\mathrm{~m}$ & $f \mathrm{~m}\left(B_{1}\right) \| \mathrm{H}$ \\
\hline 621 & $\mathrm{sh}$ & $\mathrm{M}\left(A_{1}\right) \| \mathrm{C}$ & 1,048 & $\mathrm{w}$ & $\mathrm{M}\left(A_{1}\right) \| \mathrm{H}$ \\
\hline 651 & vW & $\mathrm{m}\left(A_{1}\right) \| \mathrm{C}$ & $\begin{array}{l}1,078 \\
1,090\end{array}$ & $\begin{array}{l}\mathrm{m} \\
\mathrm{m}\end{array}$ & \} $\mathrm{m}\left(A_{1}\right) \| \mathrm{H}$ \\
\hline 693 & vs & $\mathrm{m}\left(B_{2}\right), \mathrm{M}\left(B_{2}\right) \perp \mathrm{C}$ & 1,159 & $\mathrm{w}$ & $\mathrm{M}\left(B_{1}\right) \| \mathrm{H}$ \\
\hline 724 & vs & $\mathrm{M}\left(B_{2}\right)$ & 1,172 & $\mathrm{w}$ & $\mathrm{m}\left(B_{1}\right) \mathrm{H}$ \\
\hline 780 & vs & & 1,183 & $\mathrm{w}$ & $\mathrm{m}\left(B_{1}\right) \| \mathrm{H}$ \\
\hline 791 & $\mathrm{~s}$ & $\mathrm{M}\left(B_{2}\right) \perp \mathrm{H}$ & & & \\
\hline $\begin{array}{l}807 \\
810\end{array}$ & $\begin{array}{l}\mathrm{m} \\
\mathrm{m}\end{array}$ & & & & \\
\hline
\end{tabular}

TABLE 1. Frequencies and assignments of some bands in the infrared spectra of polyphenyls - Continued

\begin{tabular}{|c|c|c|c|c|c|}
\hline \multicolumn{6}{|c|}{ 1-(3-xenyl)-3-(4-xenyl) benzene } \\
\hline $\begin{array}{r}\mathrm{cm}^{-1} \\
392 \\
403 \\
419 \\
434 \\
482\end{array}$ & $\begin{array}{l}\mathrm{vw} \\
\mathrm{m} \\
\mathrm{s} \\
\mathrm{W} \\
\mathrm{W} \\
\\
\mathrm{W} \\
\mathrm{vs} \\
\mathrm{vs} \\
\mathrm{vs} \\
\mathrm{s}\end{array}$ & $\begin{array}{l}\left\{\mathrm{p}(A \mathrm{u}), \mathrm{m}\left(A_{2}\right),\right. \\
\mathrm{m}\left(A_{2}\right) \perp \mathrm{C} \\
\mathrm{p}\left(B_{1}=\right) \perp \mathrm{C} \\
\mathrm{m} \\
\mathrm{m} \\
\mathrm{M}\left(B_{1}\right), \mathrm{m}\left(B_{1}\right) \| \mathrm{C} \\
\mathrm{M}\left(A_{1}\right) \| \mathrm{C} \\
\mathrm{m}\left(A_{1}\right) \| \mathrm{C} \\
\mathrm{m}\left(A_{1}\right) \| \mathrm{C} \\
\mathrm{M}\left(B_{2}\right), \mathrm{m}\left(B_{2}\right), \\
\mathrm{p}\left(B_{3 \mathrm{~g}}\right) \perp \mathrm{C} \\
\mathrm{M}\left(B_{2}\right) \perp \mathrm{H}\end{array}$ & $\begin{array}{r}c m^{-1} \\
782 \\
801 \\
838 \\
848 \\
882 \\
913 \\
972 \\
1,008 \\
1,014 \\
1,042 \\
1,081 \\
1,138 \\
1,161\end{array}$ & $\begin{array}{l}\mathrm{S} \\
\mathrm{m} \\
\mathrm{S} \\
\mathrm{S} \\
\mathrm{S} \\
\mathrm{W} \\
\mathrm{W} \\
\mathrm{W} \\
\mathrm{W} \\
\mathrm{W} \\
\mathrm{W} \\
\mathrm{W} \\
\mathrm{W} \\
\mathrm{W} \\
\mathrm{W}\end{array}$ & $\begin{array}{l}\left\{\mathrm{m}\left(B_{2}\right) \perp \mathrm{H}\right. \\
\mathrm{p}\left(B_{1 \mathrm{u}}\right), \mathrm{p}\left(B_{2 \mathrm{~g}}\right) ? \perp \mathrm{H} \\
\mathrm{m}\left(B_{2}\right) \perp \mathrm{H} \\
\mathrm{M}\left(B_{2}\right), \mathrm{m}\left(A_{2}\right), \\
\mathrm{p}\left(A_{\mathrm{u}}, B_{3 \mathrm{~g}}\right) \perp \mathrm{H} \\
\mathrm{m}\left(B_{2}\right) \perp \mathrm{H} \\
\mathrm{m}\left(B_{2}\right), \mathrm{p}\left(B_{2 \mathrm{u}}\right), \\
\mathrm{M}\left(A_{1}\right) \| \mathrm{C} \\
\mathrm{p}\left(B_{2}\right) \mathrm{H} \\
\mathrm{m}\left(B_{1}\right), \mathrm{M}\left(A_{1}\right) \| \mathrm{H} \\
\mathrm{m}\left(A_{1}\right), \mathrm{M}\left(B_{1}\right) \| \mathrm{H} \\
\mathrm{p}\left(B_{3} \mathrm{u}\right) \| \mathrm{H} \\
\mathrm{m}\left(B_{1}\right), \mathrm{p}\left(A_{\mathrm{g}}\right), \\
\mathrm{M}\left(B_{1}\right) \| \mathrm{H} \\
\mathrm{m}\left(B_{1}\right), \mathrm{p}\left(B_{1 \mathrm{~g}}\right) \| \mathrm{H}\end{array}$ \\
\hline \multicolumn{6}{|c|}{$m$-Sexaphenyl } \\
\hline $\begin{array}{l}402 \\
436 \\
480 \\
514 \\
577 \\
613 \\
629 \\
698 \\
690 \\
698\end{array}$ & $\begin{array}{c}\mathrm{m} \\
\mathrm{m} \\
\mathrm{w} \\
\mathrm{w} \\
\mathrm{vw} \\
\mathrm{s} \\
\mathrm{s} \\
\mathrm{w} \text { sh } \\
\text { vw } \\
\text { w } \\
\text { vs } \\
\text { w } \\
\text { vs } \\
\text { S } \\
\text { vs } \\
\text { w } \\
\text { w }\end{array}$ & $\begin{array}{l}\mathrm{M}\left(A_{2}\right), \mathrm{m}\left(A_{2}\right) \perp \mathrm{C} \\
\mathrm{m} \\
\mathrm{M}\left(B_{2}\right) \perp \mathrm{C} \\
\mathrm{m} \\
\mathrm{m} \\
\mathrm{M}\left(B_{1}\right), \mathrm{m}\left(B_{1}\right) \| \mathrm{C} \\
\mathrm{M}\left(A_{1}\right) \| \mathrm{C} \\
\mathrm{M}\left(A_{1}\right) \| \mathrm{C} \\
\mathrm{M}\left(B_{2}\right), \mathrm{m}\left(B_{2}\right) \perp \mathrm{C} \\
\mathrm{M}\left(B_{2}\right) \perp \mathrm{H} \\
\mathrm{m}\left(B_{2}\right) \perp \mathrm{H}\end{array}$ & $\begin{array}{r}873 \\
883 \\
897 \\
907 \\
913 \\
\\
970 \\
1,013 \\
1,034 \\
1,058 \\
1,070 \\
1,079 \\
1,094 \\
1,158 \\
1,180\end{array}$ & $\begin{array}{c}\mathrm{s} \\
\mathrm{s} \\
\mathrm{m} \\
\mathrm{w} \\
\mathrm{w} \\
\mathrm{w} \\
\mathrm{W} \\
\mathrm{m} \\
\mathrm{w} \\
\mathrm{w} \\
\mathrm{w} \\
\mathrm{m} \\
\mathrm{m} \\
\mathrm{w} \\
\mathrm{w} \\
\mathrm{w}\end{array}$ & $\begin{array}{l}\int \mathrm{m}\left(B_{2}\right) \perp \mathrm{H} \\
\mathrm{m}\left(A_{2}\right), \mathrm{M}\left(B_{2}\right) \perp \mathrm{H} \\
\mathrm{m}\left(B_{2}\right) \perp \mathrm{H} \\
\mathrm{M}\left(A_{1}\right), \mathrm{m}\left(A_{1}\right) \| \mathrm{C} \\
\mathrm{M}\left(A_{1}\right) \| \mathrm{H} \\
\mathrm{m}\left(B_{1}\right) \| \mathrm{H} \\
\mathrm{m}\left(A_{1}\right) \| \mathrm{H} \\
\mathrm{M}\left(B_{1}\right) \| \mathrm{H} \\
\mathrm{m}\left(B_{1}\right), \mathrm{M}\left(B_{1}\right) \| \mathrm{H} \\
\mathrm{m}\left(B_{1}\right) \| \mathrm{H}\end{array}$ \\
\hline \multicolumn{6}{|c|}{$m$-Octaphenyl } \\
\hline $\begin{array}{l}758 \\
772 \\
728 \\
791 \\
802 \\
810\end{array}$ & $\begin{array}{c}\mathrm{m} \\
\mathrm{m} \\
\mathrm{w} \\
\mathrm{m} \\
\mathrm{vw} ? \\
\mathrm{~s} \\
\mathrm{w} \\
\mathrm{vs} \\
\mathrm{vs} \\
\mathrm{vs} \\
\mathrm{vs} \\
\mathrm{vs} \\
\mathrm{vs} \\
\mathrm{ssh} \\
\mathrm{s} \\
\mathrm{w} \text { sh }\end{array}$ & $\begin{array}{l}\mathrm{M}\left(A_{2}\right), \mathrm{m}\left(A_{2}\right) \perp \mathrm{C} \\
\mathrm{m}\left(B_{2}\right) \perp \mathrm{C} \\
\mathrm{m} \\
\mathrm{m} \\
\mathrm{m}\left(B_{1}\right), \mathrm{M}\left(B_{1}\right) \| \mathrm{C} \\
\mathrm{m}\left(A_{1}\right) \| \mathrm{C} \\
\mathrm{M}\left(B_{2}\right), \mathrm{m}\left(B_{2}\right) \perp \mathrm{C} \\
\mathrm{M}\left(B_{2}\right) \perp \mathrm{H}\end{array}$ & $\begin{array}{r}875 \\
887 \\
897 \\
914 \\
922 \\
\\
975 \\
1,015 \\
1,031 \\
1,070 \\
1,076 \\
1,093 \\
1,157 \\
1,174 \\
1,181\end{array}$ & $\begin{array}{c}\mathrm{s} \\
\mathrm{s} \\
\mathrm{sh} \\
\mathrm{m} \\
\mathrm{vw} \mathrm{sh} \\
\\
\mathrm{w} \\
\mathrm{m} \\
\mathrm{w} \\
\mathrm{w} \\
\mathrm{m} \\
\mathrm{m} \\
\mathrm{w} \\
\mathrm{w} \\
\mathrm{w}\end{array}$ & $\left\{\begin{array}{l}\mathrm{m}\left(B_{2}\right) \perp \mathrm{H} \\
\mathrm{m}\left(A_{2}\right) \perp \mathrm{H} \\
\mathrm{m}\left(B_{2}\right) \perp \mathrm{H} \\
\mathrm{M}\left(A_{1}\right), \mathrm{m}\left(A_{1}\right) \| \mathrm{C} \\
\mathrm{m}\left(B_{1}\right), \mathrm{M}\left(A_{1}\right) \| \mathrm{H} \\
\mathrm{M}\left(B_{1}\right) \| \mathrm{H} \\
\begin{array}{l}\mathrm{m}\left(A_{1}\right) \| \mathrm{H} \\
\mathrm{M}\left(B_{1}\right) \| \mathrm{H} \\
\mathrm{m}\left(B_{1}\right) \| \mathrm{H}\end{array}\end{array}\right.$ \\
\hline
\end{tabular}

0.001-in. spacer and with no spacer for the thin layer. For spectra in the rocksalt region, $2 \mathrm{mg}$ and $0.25 \mathrm{mg}$ of sample were suspended in $100 \mathrm{mg}$ of potassium iodide; the lower concentration was necessary to resolve the strong bands in the $13-$ to $15-\mu$ region. For the cesium bromide region $5 \mathrm{mg}$ of sample in 150 $\mathrm{mg}$ of potassium iodide was required in order to give observable spectra. The mineral oil mull technique is not satisfactory for the observation of spectra of crystalline polyphenyls because of the solubility of these materials in paraffins. The spectrum of biphenyl in mineral oil, for example, resembles that of a carbon tetrachloride solution.

Two obviously different spectra are presented for $m$-quatraphenyl. The samples, obtained from different sources, were evidently of different crystal structure. Spectra of liquid films of the two samples 
were identical, as were spectra of crystalline films deposited on rocksalt windows by cooling the melted material. After making due allowances for the effect of orientation [8] on the band intensities, it seems that the spectra of the crystalline films most nearly resemble the pellet spectrum of $m$-quatraphenyl-II. A specimen of $m$-quatraphenyl was melted in vacuum and recrystallized. The spectrum of the resulting material was that of $m$-quatraphenyl-II. When the same substance was dissolved in benzene and recrystallized by slow evaporation, a spectrum of $m$-quatraphenyl-I was obtained. The spectrum of $m$-quatraphenyl-II shown in figures 3 and 4 evidently contains some $m$-quatraphenyl-I. In some preparations the bands at 784 and $880 \mathrm{~cm}^{-1}$, for example, were greatly weakened. The possibility that rotational isomerism might be responsible for the two forms was investigated by studying the effect of temperature on the spectrum of a melted film. A cell was constructed for use at temperatures as high as $250^{\circ} \mathrm{C}$. Spectra were observed between 2 and $15 \mu$ with a Beckman IR-4 spectrophotometer equipped with sodium chloride prisms. The results of this study are discussed in section 4 .

The polyphenyls measured were obtained from different sources. Biphenyl, and $o_{-}, m$-, and $p$-terphenyl were Eastman chemicals which had been purified by recrystallization. The higher $m$-polyphenyls, viz, quatraphenyl-I, quinquaphenyl, hexaphenyl, and octaphenyl were prepared in this laboratory by R. L. Alexander, Jr. [9]. The samples of $o$ - and $p$-quatraphenyl were purchased from the Organic Specialties Co., and the sample marked $m$ quatraphenyl-II was bought from the Mathieson Co. The remaining five compounds (quatra- and quinquaphenyls) were obtained from the laboratory of Prof. G. F. Woods at the University of Marvland. Their preparation and properties have been reported in the literature $[10,11,12]$.

The spectrum of $p$-quatraphenyl contains weak bands at 740 and $714 \mathrm{~cm}^{-1}$ not observed in the spectrum given by Cannon and Sutherland [13] although the spectra seem to agree in other respects. These bands are presumably contributed by impurities.

\section{Discussion}

Monosubstituted and ortho- and metadisubstituted rings have pseudosymmetry $C_{2 \mathrm{v}}$, while paradisubstituted rings have pseudosymmetry $V_{\mathrm{h}}$. The symmetry species of the vibrations of the substituted rings correlate with those of benzene as shown in table 2. Vibrations that are forbidden to appear in the infrared spectra under the selection rules appropriate for a given symmetry group are shown in parentheses. They are actually quite likely to appear in the spectra, though probably with reduced intensity. The vibrations listed in table 2 and discussed in the following paragraphs are arranged on the basis of an approximate description, e. g., C-H stretching, solely for convenience. It is recognized that more than one symmetry coordinate is involved in most normal vibrations. It should also be noted
TABLE 2. Correlation of symmetry species of substituted rings ${ }^{\text {a }}$

\begin{tabular}{|c|c|c|c|c|c|c|}
\hline \multirow[b]{2}{*}{$\begin{array}{l}\text { para }\left(V_{\mathrm{h}}\right) \\
\text { meta }\left(C_{2 \mathrm{v}}\right) \\
\text { ortho }\left(C_{2 \mathrm{v}}\right) \\
\text { mono }\left(C_{2 \mathrm{v}}\right) \\
\text { benzene }\left(D_{6 \mathrm{~h}}\right)\end{array}$} & \multicolumn{3}{|c|}{$\begin{array}{l}\text { Out-of-plane } \mathrm{C}-\mathrm{C} \\
\text { bending }\end{array}$} & \multicolumn{3}{|c|}{ In-plane $\mathrm{C}-\mathrm{C}$ bending } \\
\hline & $\begin{array}{l}\left(B_{3 \mathrm{~g}}\right) \\
B_{2} \\
\left(A_{2}\right) \\
B_{2} \\
B_{2 \mathrm{~g}}\end{array}$ & $\begin{array}{l}\left(A_{\mathrm{u}}\right) \\
\left(A_{2}\right) \\
\left(A_{2}\right) \\
\left(A_{2}\right) \\
\quad\left(E_{2 \mathrm{u}}\right)\end{array}$ & $\begin{array}{l}B_{1 \mathrm{u}} \\
B_{2} \\
B_{2} \\
B_{2}\end{array}$ & $\begin{array}{l}\left(A_{\mathrm{g}}\right) \\
A_{1} \\
A_{1} \\
A_{1} \\
\quad\left(E_{2 \mathrm{~g}}\right)\end{array}$ & $\begin{array}{c}\left(B_{1 \mathrm{~g}}\right) \\
B_{1} \\
B_{1} \\
B_{1}\end{array}$ & $\begin{array}{l}B_{2 \mathrm{u}} \\
A_{1} \\
B_{1} \\
A_{1} \\
\left(B_{1 \mathrm{u}}\right)\end{array}$ \\
\hline
\end{tabular}

Out-of-plane $\mathrm{C}-\mathrm{H}$ bending

\begin{tabular}{|c|c|c|c|c|c|}
\hline $\begin{array}{l}\left(B_{3 \mathrm{~g}}\right) \mathrm{S} \\
B_{2} \mathrm{~S} \\
\left(A_{2}\right) \mathrm{S} \\
B_{2} \mathrm{~S} \\
\left(B_{2 \mathrm{~g}}\right)\end{array}$ & $\begin{array}{l}\left(B_{2 \mathrm{~g}}\right) \\
\left(A_{2}\right) \\
\left(A_{2}\right) \\
\left(A_{2}\right){ }_{\left(E_{1 \mathrm{~g}}\right)}\end{array}$ & $\begin{array}{l}\left(B_{3 \mathrm{~g}}\right) \\
B_{2} \\
B_{2} \\
B_{2}\end{array}$ & $\begin{array}{l}B_{1 \mathrm{u}} \\
B_{2} \\
B_{2} \\
B_{2} \\
A_{2 \mathrm{u}}\end{array}$ & 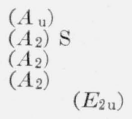 & $\begin{array}{l}B_{1 \mathrm{u}} \mathrm{S} \\
B_{2} \\
B_{2} \mathrm{~S} \\
B_{2}\end{array}$ \\
\hline
\end{tabular}

\begin{tabular}{|c|c|c|c|c|c|}
\hline \multicolumn{6}{|c|}{ In-plane $\mathrm{C}-\mathrm{H}$ bending } \\
\hline $\begin{array}{c}\left(B_{1 \mathrm{~g}}\right) \\
B_{1} \\
B_{1} \\
B_{1} \\
\left(A_{2 \mathrm{~g}}\right)\end{array}$ & $\begin{array}{l}\left(A_{\mathrm{g}}\right) \\
A_{1} \mathrm{~S} \\
A_{1} \mathrm{~S} \\
A_{1} \quad\left(E_{2 \mathrm{~g}}\right)\end{array}$ & $\begin{array}{l}\left(B_{1 \mathrm{~g}}\right) \mathrm{S} \\
B_{1} \\
B_{1} \\
B_{1}\end{array}$ & $\begin{array}{l}B_{3 \mathrm{u}} \\
B_{1} \\
A_{1} \\
B_{1} \\
\left(B_{2 \mathrm{u}}\right)\end{array}$ & $\begin{array}{ll}B_{2 \mathrm{u}} & \\
A_{1} & \\
A_{1} & \\
A_{1} & \\
& E_{1 \mathrm{u}}\end{array}$ & $\begin{array}{l}B_{3 \mathrm{u}} \mathrm{S} \\
B_{1} \mathrm{~S} \\
B_{1} \mathrm{~S} \\
B_{1} \mathrm{~S}\end{array}$ \\
\hline \multicolumn{6}{|c|}{$\mathrm{C}-\mathrm{C}$ stretching } \\
\hline $\begin{array}{l}\left(A_{\mathrm{g}}\right) \\
A_{1} \\
A_{1} \\
A_{1} \\
\left(A_{1 \mathrm{~g}}\right)\end{array}$ & $\begin{array}{ll}\left(A_{\mathrm{g}}\right) & \\
A_{1} & \\
A_{1} & \\
A_{1} & \\
& \left(E_{2 \mathrm{~g}}\right)\end{array}$ & $\begin{array}{l}\left(B_{\mathrm{g}}\right) \\
B_{1} \\
B_{1} \\
B_{1}\end{array}$ & $\begin{array}{l}B_{3 \mathrm{u}} \\
B_{1} \\
A_{1} \\
B_{1} \\
\left(B_{2} \mathrm{u}\right)\end{array}$ & $\begin{array}{ll}B_{2 \mathrm{u}} & \\
A_{1} & \\
A_{1} & \\
A_{1} & \\
& E_{1 \mathrm{u}}\end{array}$ & $\begin{array}{l}B_{3 \mathrm{u}} \\
B_{1} \\
B_{1} \\
B_{1}\end{array}$ \\
\hline \multicolumn{6}{|c|}{$\mathrm{C}-\mathrm{H}$ stretching } \\
\hline $\begin{array}{l}\left(A_{\mathrm{g}}\right) \mathrm{S} \\
A_{1} \mathrm{~S} \\
A_{1} \mathrm{~S} \\
A_{1} \mathrm{~S} \\
\left(A_{1 \mathrm{~g}}\right)\end{array}$ & $\begin{array}{ll}\left(A_{\mathrm{g}}\right) & \\
A_{1} & \\
A_{1} & \\
A_{1} & \\
& \end{array}$ & $\begin{array}{l}\left(B_{\mathrm{gg}}\right) \\
B_{1} \\
B_{1} \\
B_{1}\end{array}$ & $\begin{array}{c}B_{2 \mathrm{u}} \\
A_{1} \\
B_{1} \\
A_{1} \\
\left(B_{1 \mathrm{u}}\right)\end{array}$ & $\begin{array}{l}B_{2 \mathrm{u}} \mathrm{S} \\
A_{1} \\
A_{1} \\
A_{1} \quad \\
\quad E_{1 \mathrm{u}}\end{array}$ & $\begin{array}{l}B_{3 \mathrm{u}} \\
B_{1} \mathrm{~S} \\
B_{1} \mathrm{~S} \\
B_{1}\end{array}$ \\
\hline
\end{tabular}

a Species in parentheses are infrared inactive under strict selection rules. S designates vibrations involving carbon-to-substituent bonds, rather than carbonto-hydrogen bonds.

that the stretching and bending of carbon-tosubstituent bonds in the substituted aromatics correlate with stretching and bending of carbon-tohydrogen bonds in benzene, requiring adjustments in the counting of vibrations.

\subsection{C - C Stretching Vibrations}

Characteristic aromatic ring stretching vibrations are found near 1,600 and 1,450 to $1,500 \mathrm{~cm}^{-1}$. Vibrations of ortho-, meta-, and monosubstituted rings that correlate with the degenerate $E_{2 g}$ Raman line of benzene at $1,595 \mathrm{~cm}^{-1}$ are infrared active, but the corresponding vibrations of $V_{\mathrm{h}}$ parasubstituted rings are inactive. All of the polyphenyls have absorptions near $1,600 \mathrm{~cm}^{-1}$ which are resolved into at least two components. Following $\mathrm{RW}$, the high-frequency component probably contains the $A_{1}$ absorptions, and the low-frequency component the $B_{1}$ absorptions of the mono-, ortho-, and metasubstituted rings. The $1,600 \mathrm{~cm}^{-1}$ bands are quite weak in $p$-terphenyl and even weaker in $p$-quatraphenyl, as would be expected with a reduction in the mono- to pararing ratio. However, there seems to be no noticeable weakening of the $1,600 \mathrm{~cm}^{-1}$ bands in the other polyphenyls which contain parasubstituted rings. All of the polyphenyl vibrations that are correlated with the $E_{1 u}$ band of benzene at 
$1,485 \mathrm{~cm}^{-1}$ are infrared active, and the spectra of all of the polyphenyls have a complex series of bands in this region. $\mathrm{RW}$ have assigned the bigher frequency absorptions near $1,500 \mathrm{~cm}^{-1}$ to the $A_{1}$ modes in mono-, ortho-, and metasubstituted rings and the $B_{2 \mathrm{u}}$ mode in parasubstituted rings; and they assigned the lower frequency absorptions near $1,450 \mathrm{~cm}^{-1}$ to the $B_{1}$ modes in mono-, ortho-, and metasubstituted rings and the $B_{3 \mathrm{u}}$ mode in parasubstituted rings. Those polyphenyls that contain meta- and parasubstituted rings have bands near $1,400 \mathrm{~cm}^{-1}$ which are missing or weak in compounds containing a high proportion of orthosubstituted rings and in biphenyl. These bands are probably $\mathrm{C}-\mathrm{C}$ stretching modes shifted from the 1,150 to $1,500-\mathrm{cm}^{-1}$ region, possibly because of the influence of neighboring rings. These bands are discussed further in section 3.6.

The $\mathrm{C}-\mathrm{C}$ vibrations correlated with the $A_{1 \mathrm{~g}}$ Raman line of benzene at $992 \mathrm{~cm}^{-1}$ are sensitive to substituents and have not been assigned. Vibra. tions corresponding to the uncertain $B_{2 u}$ benzene vibration have been placed at 1,324 and $1,315 \mathrm{~cm}^{-1}$ (both weak) in mono- and orthosubstituted ring compounds by $\mathrm{RW}$. Weak absorptions are observed near there in the infrared spectra of the polyphenyls.

\subsection{In-Plane Ring Bending Vibrations}

The doubly degenerate $E_{2 \mathrm{~g}}$ ring bending vibration of benzene is found at $605 \mathrm{~cm}^{-1}$ in the Raman spectrum. The $A_{1}$ and $B_{1}$ pair of vibrations in mono-, ortho-, and metasubstituted rings which correlate with the $E_{2 \mathrm{~g}}$ benzene mode should be active in the infrared, but the corresponding $A_{\mathrm{g}}$ and $B_{1 \mathrm{~g}}$ modes of parasubstituted rings should be inactive under $V_{\mathrm{h}}$ selection rules. All of the polyphenyls studied have a band at $614 \pm 4 \mathrm{~cm}^{-1}$. This band, which is fairly strong, except in the case of $p$-terphenyl and $p$ quatraphenyl, is assigned to the $B_{1}$ modes of mono-, ortho-, and metasubstituted rings. RW place the corresponding $B_{1 \mathrm{~g}}$ mode of parasubstituted rings at $699 \pm 6 \mathrm{~cm}^{-1}$ in the region obscured by strong absorption of the monosubstituted rings. All but three of the polyphenyls absorb weakly at $625 \pm 6$ $\mathrm{cm}^{-1}$. These bands are assigned to the $A_{1}$ mode of monosubstituted rings placed at $620 \pm 4(618 \pm 5$ $\mathrm{cm}^{-1}$ in the Raman effect) by $\mathrm{RW}$. In addition, all but two of the compounds containing metasubstituted rings have bands at $647 \pm 11 \mathrm{~cm}^{-1}$ which we assign to the $A_{1}$ mode of metasubstituted rings.

The $B_{1 \mathrm{u}}$ ring deformation of benzene has been calculated by Lord and Andrews [14] to lie at 1,008 $\mathrm{cm}^{-1}$. RW assign bands at $1,001 \pm 4$ and $999 \pm 5$ $\mathrm{cm}^{-1}$ to the corresponding $A_{1}$ modes of mono- and metasubstituted rings, respectively. All of the polyphenyls have bands of medium intensity near $1,000 \mathrm{~cm}^{-1}$ which probably represent these modes as well as the corresponding $B_{1}$ modes in ortho- and $B_{2 u}$ modes in parasubstituted rings.

\subsection{In-Plane $\mathrm{C}-\mathrm{H}$ Bending Vibrations}

The in-plane $\mathrm{C}-\mathrm{H}$ bending vibrations of benzene lie in the fairly narrow region from about 1,030 to $1,200 \mathrm{~cm}^{-1}$. Of these, a band is observed at 1,037 $\mathrm{cm}^{-1}$ in the infrared and another at $1,178 \mathrm{~cm}^{-1}$ in the Raman spectrum, while the remaining two inactive modes have been calculated [14] to lie at 1,190 and $1,145 \mathrm{~cm}^{-1}$. After removing the carbonto-substituent bond bending modes there remain $1 A_{1}$ and $3 B_{1} ; 2 A_{1}$ and $2 B_{1} ; 1 A_{1}$ and $3 B_{1}$; and 1 $B_{3 \mathrm{u}}, 1 B_{2 \mathrm{u}}, 1 B_{1 \mathrm{~g}}$, and $1 A_{\mathrm{g}}$ transitions in the mono-, ortho-, meta-, and parasubstituted rings, respectively. Only the $B_{2 \mathrm{u}}$ and $B_{3 \mathrm{u}}$ transitions are infrared active in parasubstituted rings under $V_{\mathrm{h}}$ selection rules.

RW have observed that numerous bands in the 1,000 - to $1,200-\mathrm{cm}^{-1}$ region are relatively invariant from molecule to molecule of a given isomeric type. They assign $1,029 \pm 5,1,031 \pm 5,1,045 \pm 7$, and $1,018 \pm 10 \mathrm{~cm}^{-1}$ to $A_{1}$ mono, $A_{1}$ ortho, $B_{1}$ meta, and $B_{2 u}^{2}$ paravibrations; $1,072 \pm 7,1,126 \pm 12,1,081 \pm 10$, and $1,125 \pm 10 \mathrm{~cm}^{-1}$ to $B_{1}$ mono, $B_{1}$ ortho, $A_{1}$ meta, and $B_{3 \mathrm{u}}$ paravibrations; and $1,156 \pm 5,1,159 \pm 7,1,165 \pm 6$, and $1,179 \pm 11 \mathrm{~cm}^{-1}$ to $B_{1}$ mono, $A_{1}$ ortho, $B_{1}$ meta, and $A_{\mathrm{g}}$ paravibrations, respectively. These three groups of bands are readily associated with bands found near $1,040,1,080$, and $1,160 \mathrm{~cm}^{-1}$, respectively, in all of the polyphenyl spectra. RW further assign a band at $1,177 \pm 6 \mathrm{~cm}^{-1}$ to an $A_{1}$ mode of monosubstituted benzenes. Bands found near 1,190 $\mathrm{cm}^{-1}$ in the polyphenyl spectra very likely correspond to this $A_{1}$ monomode, and also to the $B_{1}$ ortho, $B_{1}$ meta, and the forbidden $B_{1 \mathrm{~g}}$ paravibrations as well.

The regions of absorption, as given by $\mathrm{RW}$, are seen to depend somewhat on isomeric substitution, and lead to some complexity in the polyphenyl bands. For example, the spectrum of $p$-terphenyl has a pair of bands with peaks at 1,043 and 1,028 $\mathrm{cm}^{-1}$, which can be assigned to the $A_{1}$ mono and $B_{2 u}$ paravibrations, respectively. These separations are more than the amount expected from RW's tables, perhaps because of interactions of the Fermi type.

\subsection{Out-of-Plane $\mathrm{C}-\mathrm{H}$ Bending Vibrations}

These are the vibrations used most frequently for the qualitative analysis of substituted aromatic compounds. RW give the following regions of absorption for the strong bands: Mono $B_{2} 751 \pm 15$; ortho $\bar{B}_{2} 751 \pm 7$; meta $B_{2} 782 \pm 9$, and $\bar{B}_{2} 876 \pm 10$; and para $B_{1 \mathrm{u}} 817 \pm 13 \mathrm{~cm}^{-1}$. These values do not hold for strongly electrophylic substituents such as fluorine and nitro groups. In the spectra of polyphenyls the metabands are found at $795 \pm 13$ and $894 \pm 21 \mathrm{~cm}^{-1}$ and the paraband at $854 \pm 6 \mathrm{~cm}^{-1}$. These values are somewhat higher than those given by RW. The orthobands are also found at higher frequencies, $776 \pm 9 \mathrm{~cm}^{-1}$, rendering them distinct, or nearly so, from the monobands at $766 \pm 22 \mathrm{~cm}^{-1}$.

The weaker $\mathrm{C}-\mathrm{H}$ out-of-plane bending vibrations are given by $\mathrm{RW}$ as follow: Mono $B_{2} 908 \pm 10, B_{2}$ and $A_{2}, 962 \pm 6$, and $982 \pm 6, A_{2} 837 \pm 10$; ortho $A_{2} 865 \pm 14$ and $977 \pm 9, B_{2} 934 \pm 11 ;$ meta $A_{2} 904$ $\pm 13, B_{2} 964 \pm 10$; and para $B_{2 \mathrm{~g}} 840$ (in the Raman effect), $A_{\mathrm{u}}$ or $\vec{B}_{3 \mathrm{~g}} 961 \pm 12 \mathrm{~cm}^{-1}$. All of the polyphenyls have one or more weak-to-medium bands near $850 \mathrm{~cm}^{-1}$ which are easily mistaken for the 
characteristic $B_{1 \mathrm{u}}$ para-absorption. It is likely that these bands are contributed by vibrations involving the connecting links (see section 3.6).

A more careful study of the strong characteristic $\mathrm{C}-\mathrm{H}$ bands reveals some curious progressions. The metabands are seen to be resolved into components with increasing complexity in compounds containing larger numbers of such rings. In those cases where only one metasubstituted ring is present the strongest peak in the $894 \mathrm{~cm}^{-1}$ region is generally found at a somewhat higher frequency than when more than one metaring is present. In the $795-\mathrm{cm}^{-1}$ region (see fig. 9) the strongest peak shifts regularly to lower frequencies as the number of metarings increases, while an increasingly complex set of weaker peaks develops on the high-frequency side of the major peak. This complex set of weaker peaks develops on the high-frequency side of the major peak. This complex structure is less resolved in the spectra of some of the polyphenyls in the solution phase, but it is still recognizable, thus eliminating the possibility that lattice vibrations or intermolecular vibrational interactions are involved. A plausible explanation is that the $\mathrm{C}-\mathrm{H}$ bending vibrations of different metasubstituted rings in the same molecule are interacting. This idea can be developed further through the use of quantum mechanical perturbation theory, which shows that if all interaction terms are set equal to zero except those between nearest neighbors, which are assumed to be identical, the degeneracy of the vibrations is removed and the levels are split symmetrically. The inclusion of next-nearest neighbor interactions results in a regular change in the separation of adjacent components with the spacing of the bands becoming compressed at one side and expanded at the other.

The magnitude of the interaction force constant

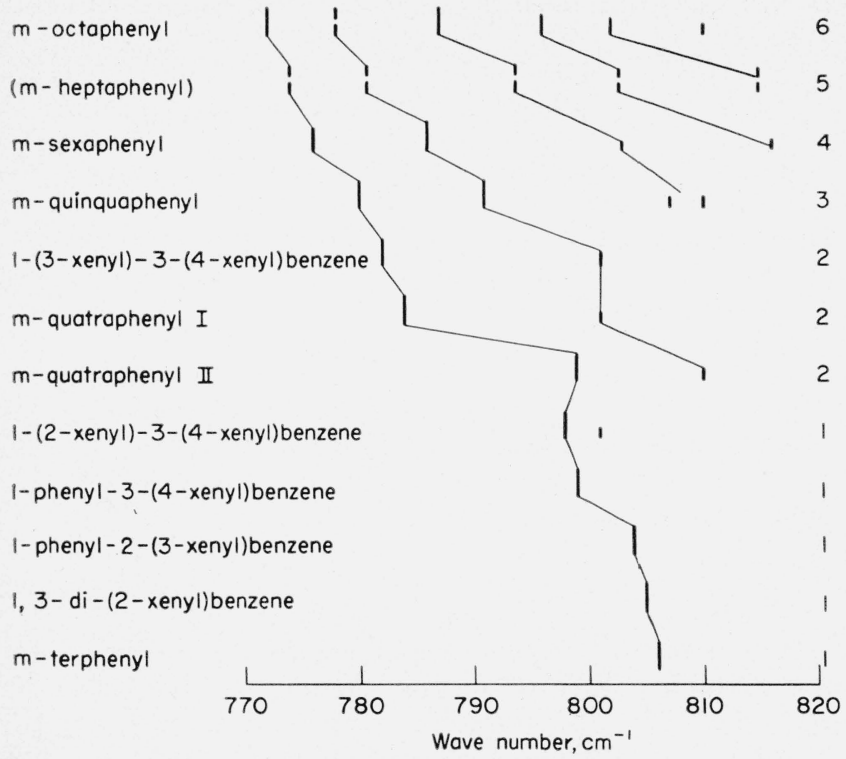

FIgURE 9. Bands in 800-cm-1 region for compounds containing metasubstituted rings.

Numbers at right indicate number of metasubstituted rings in the molecule. $m$-Heptaphenyl was not available for study, but predicted bands are included in the figure. The line predicted for $m$-octaphenyl at $780 \mathrm{~cm}^{-1}$, was not observed, perhaps because of insufficient resolution. must be about 0.2 of the value of the diagonal terms in order to explain the magnitude of the observed splitting. The relative intensities of the components of the bands were determined from the elements of the dipole moment transition matrices after first determining the correct sums of the product functions for each value of the perturbation energy. The theory predicts that the component of lowest frequency will be the strongest, with the intensities of the higher frequency components decreasing more or less regularly in agreement with the observed spectra, provided that $\lambda$, the interaction force constant, is taken to be negative. The physical significance of a negative value is that it is easier to distort the hydrogens on a ring out of their normal plane if the hydrogens on the neighboring ring are already out of their plane. It is interesting to observe that Kakiuti and Shimanouchi [6] calculate negative values for the out-of-plane interaction force constants of neighboring hydrogens on the same ring, and that the magnitude of the interaction constant is about 0.18 that of the $\mathrm{C}-\mathrm{H}$ out-of-plane bending force constant.

Orr [15] has recently explained the broadening of the out-of-plane bending bands $\left(960 \mathrm{~cm}^{-1}\right)$ of the olefinic hydrogens in orthosubstituted transstilbenes on the basis of damping accompanying the transfer of vibrational energy to a motion involving the substituent groups which are quite close to the olefinic hydrogens. This situation differs from that encountered in the case of the metasubstituted polyphenyls because in the stilbenes energy is transferred between vibrational states of considerably different energy. However, Orr's process is very likely involved in the case where neighboring rings are substituted in different ways.

The bands characteristic of orthosubstitution near $776 \mathrm{~cm}^{-1}$ and the $766-\mathrm{cm}^{-1}$ band of monosubstituted rings in molecules containing orthosubstituted rings are also split into complex structures, but not in so systematic a fashion as that displayed by the metaring compounds. This is probably the result of interactions between the out-of-plane $\mathrm{C}-\mathrm{H}$ vibrations of ortho- and monosubstituted rings, as well as interactions between orthosubstituted rings. The number of peaks resolved in the $760-$ to $780-\mathrm{cm}^{-1}$ band seems excessive. For example, o-terphenyl has five peaks resolved, but contain only three rings. This is possibly a consequence of the occurrence of more than one molecule per unit cell. o-Terphenyl has four molecules in each unit cell [16]. The spectrum of 0 -terphenyl in solution in carbon disulfide does not display such pronounced splitting, for only three components are resolved. o-Quatraphenyl in carbon disulfide solution, on the other hand, has five components in the $740-$ to $790-\mathrm{cm}^{-1}$ region, just as in the solid phase.

No splitting is observed in the out-of-plane $\mathrm{C}-\mathrm{H}$ bending vibrations of the parasubstituted rings, but the band is found at a somewhat lower frequency in $p$-quatraphenyl $\left(826 \mathrm{~cm}^{-1}\right)$, which contains two parasubstituted rings, than in those molecules $\left(843 \pm 5 \mathrm{~cm}^{-1}\right)$ containing only one parasubstituted ring. 


\subsection{Out-of-Plane Ring Bending Vibrations}

The frequency of the $B_{2 \mathrm{~g}}$ out-of-plane ring bending made of benzene has been calculated [6] to be $698 \mathrm{~cm}^{-1}$. RW have placed the corresponding $B_{2}$ modes of mono- and metasubstituted rings at $697 \pm 11$ and $690 \pm 15 \mathrm{~cm}^{-1}$, respectively. These bands are quite intense in the infrared spectra and are used with the nearby $\mathrm{C}-\mathrm{H}$ bands in the qualitative analysis of substituted aromatic compounds. RW place the infrared forbidden $B_{3 \mathrm{~g}}$ vibration of parasubstituted rings at $699 \pm 6 \mathrm{~cm}^{-1}$ where weak bands are sometimes observed in the infrared spectra. The corresponding orthoband is of species $A_{2}$ and is also forbidden in the infrared.

The $404 \mathrm{~cm}^{-1} E_{2 \mathrm{u}}$ benzene vibration is doubly degenerate. Its $A_{2}$ component in monosubstituted rings, and its $A_{\mathrm{u}}$ component in parasubstituted rings, are observed in the Raman effect at $406 \pm 10$ and $404 \pm 10 \mathrm{~cm}^{-1}$ according to $\mathrm{RW}$, and sometimes occur as weak absorptions in the infrared. All of the polyphenyls absorb weakly near $400 \mathrm{~cm}^{-1}$, which suggests that the $A_{2}$ components of this vibration in ortho- and metasubstituted rings also absorb here. The $B_{2}$ component corresponding to the $E_{\mathrm{u}}$ benzene vibration is reported by $R W$ to lie in the 450 - to $500-\mathrm{cm}^{-1}$ region and to be sensitive to the nature of the substituent. The polyphenyls have bands in this region which permit some general assignments, but until Raman data are available for these compounds the situation cannot be described as satisfactorily as for the 11- to $14-\mu$ region. Biphenyl has a band at $460 \mathrm{~cm}^{-1}$, and the parasubstituted compounds have single strong bands at $471 \pm 11 \mathrm{~cm}^{-1}$, which are readily assigned to the $B_{2}$ mono- and $B_{1 \mathrm{u}}$ paravibrations. The orthosubstituted compounds have a pattern. of three bands at $503 \pm 10,532 \pm 10$, and $564 \pm 7 \mathrm{~cm}^{-1}$, while the metasubstituted compounds also have a pattern of bands in this region at $454 \pm 20,514 \pm 12$, and sometimes $563 \pm 10 \mathrm{~cm}^{-1}$. Other bands usually occur also. In table 1 these bands are merely assigned to ortho- or metasubstituted rings.

\subsection{Connecting Link Vibrations and Unassigned Bands}

Each of the ring-connecting bonds contributes 1 stretching, 1 torsion, 2 in-plane bending, and 2 out-of-plane bending internal coordinates to the normal vibrations. If these internal coordinates acted independently, without simultaneous vibration of the other internal coordinates, their vibrational frequencies would probably be well below the region of observation available in this study. However, other internal coordinates are almost certainly involved and might in fact, predominate. Without actually performing a normal coordinate determination it is not possible to guess where these vibrations are to be found.

There is little doubt that the para- and metasubstituted ring systems are planar, or nearly so, while the orthosubstituted ring systems are nonplanar because of steric hindrance. Hence the ring-connecting bonds in orthosubstituted systems must have less double bond character than in the other structures.
If the vibrations Joosely described as connecting-bond stretching actually involve large contributions from motions of the ring bonds, it would be reasonable for the frequencies to be not much lower than those of the "normal" ring vibrations, and they might indeed account for the bands observed near 1,400 $\mathrm{cm}^{-1}$ in the meta- and parasubstituted polyphenyls. The loss of double bond character in the ringconnecting bonds of the orthosubstituted systems would then explain the absence of these bands in the spectra of the orthosubstituted compounds.

Five of the polyphenyls have bands of medium intensity at $727 \pm 2 \mathrm{~cm}^{-1}$. A sixth compound has a pair of weak bands at 722 and $728 \mathrm{~cm}^{-1}$ and another compound has a weak shoulder at $714 \mathrm{~cm}^{-1}$. All of these compounds contain at least 1 metasubstituted ring, all but 1 contain 5 or more rings, and all but 1 have at least 1 metasubstituted ring which is not adjacent to a monosubstituted ring. The correlation of these bands with vibrations involving connecting links to metasubstituted rings seems reasonable.

There are other bands in the infrared spectra of the polyphenyls which are possibly associated with the ring-connecting bonds. Among these are the bands at $846 \pm 13 \mathrm{~cm}^{-1}$ mentioned previously, and the multitude of absorptions observed in the cesium bromide region, particularly those correlated with ortho- and with metasubstituted rings.

\section{Rotational Isomerism in $m$-Quatraphenyl}

The connecting bond between phenyl rings in polyphenyl molecules is stabilized by about 7 $\mathrm{kcal} / \mathrm{mole}$ [17] of resonance energy. This is sufficient double-bond character to insure planarity except in such molecules as o-terphenyl [16] where the steric repulsion is too great. On the other hand, a barrier of this magnitude is not too high to permit hindered rotation and the rapid establishment of equilibrium if more than one stable configuration exists. The structure of $m$-quatraphenyl has not been determined, but it is reasonable to assume that the planar transform is the most stable one in view of the known planarity of biphenyl, $p$-terphenyl, and $p$-quatraphenyl. The planar cisform is very likely also a stable configuration with somewhat greater free energy than the transform because of the closer proximity of the hydrogen atoms.

The intensities of all bands of liquid $m$-quatraphenyl were found to decrease reversibly as the temperature was increased, partly as a result of thermal expansion, but some bands decreased more rapidly than others. Several bands were sufficiently well resolved to permit intensity measurements. The results, shown in figure 10 , are readily interpreted [18] as evidence for the existence of rotational isomers having a free energy difference of about $250 \mathrm{cal} /$ mole. A crude calculation of the additional electrostatic repulsion energy in the cisconfiguration yields a value of this order of magnitude. Included in figure 10 are data for two pairs of bands which behave similarly as the temperature is changed. The nearly zero slope $(E<20 \mathrm{cal} / \mathrm{mole})$ for these pairs is evidence that possible perturbing influ- 


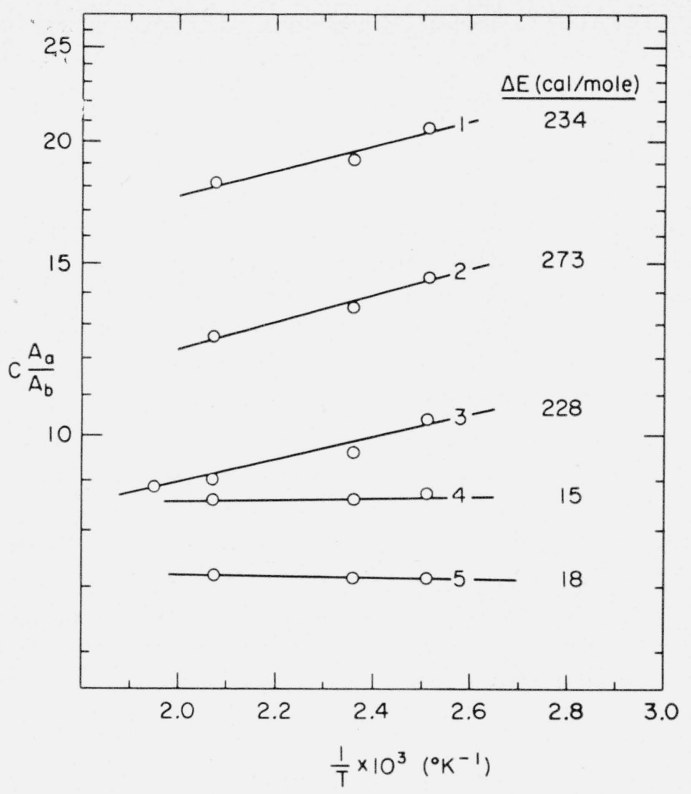

FiguRE 10. Ratios of absorbancies for several band pairs in the infrared spectrum of m-quatraphenyl as a function of temperature.

The free-energy differences are calculated from the slopes. C is an arbitrary constant, selected for each curve for convenience in plotting. Band pairs used are: $1,1,013$ and $1,075 \mathrm{~cm}^{-1} ; 2,1,094$ and $1,075 \mathrm{~cm}^{-1} ; 3,1,170$ and $1,158 \mathrm{~cm}^{-1} ; 4$ 1,170 and $1,094 \mathrm{~cm}^{-1} ; 5,1,013$ and $1,026 \mathrm{~cm}^{-1}$.

ences, such as radiation from the hot cell, are not important.

The absorptions used in this determination are in a spectral region where bands are fairly insensitive to structural changes and where contributions from mono- and metasubstituted rings overlap. This tends to reduce the sensitivity of the spectrum to changes in isomer equilibrium and could lead to a low value for the isomeric energy difference. This circumstance also renders useless one of the standard tests for the existence of isomeric forms, namely the disappearance of a set of bands upon crystallization. Consequently, this experiment failed to demonstrate whether different isomeric forms are involved in the two different crystalline samples of $m$-quatraphenyl.

Recent discoveries of the temperature sensitivity of absorption band intensities in cyclohexanone [19] and some substituted phenols [20] seem to raise questions about the validity of determining isomeric energy differences from the temperature behavior of band intensities. A completely satisfactory explanation of this behavior has not vet been given, although Liddel and Becker [21] attribute the variation of $\mathrm{O}-\mathrm{H}$ bands with temperature to alcoholsolvent interactions resembling very weak hydrogen bonds. Meanwhile, the existence of rotational isomers in liquid $m$-quatraphenyl can be considered probably established.

\section{Conclusion}

In general, the assignments given by $\mathrm{RW}$ for substituted benzenes provide a satisfactory basis for the discussion of the infrared spectra of the poly- phenyls. The assignments have been extended somewhat to include some additional vibrations, including some that are peculiar to polyphenyl compounds. The splitting observed in some of the out-of-plane bending vibration bands indicates that the individual rings of polyphenyls do not vibrate as independent units, at least as far as the out-ofplane modes are concerned. Indeed, the fine structure observed in the $\mathrm{C}-\mathrm{H}$ out-of-plane deformation vibration bands of metasubstituted rings near $790 \mathrm{~cm}^{-1}$ can be explained on the basis of interaction between a ring and its nearest neighbors. On the other hand, pronounced splitting of this sort is not observed in the case of the in-plane vibrations and many of the other out-of-plane vibrations.

It is concluded that the in-plane vibrations of polyphenyl molecules can be treated as though they they arise in individual uncoupled rings, and that the out-of-plane vibrations can also be treated in this way for classification purposes, but due allowance must be made for interactions between rings when more detailed assignments are to be made. In either case, vibrations involving the ring-connecting bonds must, of course, be treated separately.

\section{References}

[1] S. Krimm, C. Y. Liang, and G. B. B. M. Sutherland, J. Chem. Phys, 25, 549 (1956).

[2] C. Y. Liang and S. Krimm, J. Chem. Phys. 25, 563 (1956)

[3] San-Ichiro Mizushima, I. Nakagawa, and D. M. Sweeny, J. Chem. Phys. 25, 1006 (1956).

[4] L. J. Bellamy, The infrared spectra of complex molecules (John Wiley \& Sons, Inc., New York, N. Y., 1954).

[5] R. R. Randle and D. H. Whiffen, Molecular spectroscopy, Report of a Conference of the Hydrocarbon Research Group (The Institute of Petroleum, Oct. 1954).

[6] T. Shimanouchi, Y. Kakiuti, and I. Gamo, J. Chem. Phys. 25, 1245 (1956); Y. Kakiuti and T. Shimanouchi, J. Chem. Phys. 25, 1252 (1956).

[7] J. E. Stewart, J. Research NBS 54, 41 (1955) RP2563 J. Chem. Phys. 26, 248 (1957).

[8] J. E. Stewart, J. Chem. Phys. 23, 986 (1955).

[9] R. L. Alexander, Jr., J. Org. Chem. 21, 1464 (1956).

[10] G. F. Woods and I. W. Tucker, J. Am. Chem. Soc. y0, 3340 (1948)

[11] G. F. Woods and F. T. Reed, J. Am. Chem. Soc. 71, 1348 (1949)

[12] G. F. Woods, A. L. Van Artsdale, and F. T. Reed, J. Am. Chem. Soc. $\mathbf{7 \% ,} 3221(1950)$.

[13] C. G. Cannon and G. B. B. M. Sutherland, Spectrochim. Acta 4, 373 (1951).

[14] R. C. Lord and D. H. Andrews, J. Phys. Chem. 41, 149 (1937).

[15] S. F. D. Orr, Spectrochim. Acta 8, 218 (1956)

[16] J. D. H. Dounay and W. Nowachi, Crystal data, Memoir 60 (Geological Society of America, New York, N. Y., 1954).

[17] L. Pauling, The nature of the chemical bond, p. 217 (Cornell University Press, Ithaca, N. Y., 1948).

[18] San-Ichiro Mizushima, Structures of molesules and internal rotation (Academic Press, New York, N. Y. 1954).

[19] E. J. Slowinski, Jr., and G. C. Claver, J. Opt. Soc. Am. 45, 396 (1955).

[20] R. H. Hughes, R. J. Martin, and N. D. Coggeshall, J. Chem. Phys. 24, 489 (1956).

[21] U. Liddel and E. D. Becker, J. Chem. Phys. 25, 173 (1956).

Washington, August 29, 1957. 